Pure and Applied Mathematics Quarterly

Volume 1, Number 1, 68-108, 2005

\title{
Volume Growth and Curvature Decay of Positively Curved Kähler Manifolds
}

\author{
Bing-Long Chen and Xi-Ping Zhu
}

\begin{abstract}
In this paper we obtain three results concerning the geometry of complete noncompact positively curved Kähler manifolds at infinity. The first one states that the order of volume growth of a complete noncompact Kähler manifold with positive bisectional curvature is at least half of the real dimension (i.e., the complex dimension). The second one states that the curvature of a complete noncompact Kähler manifold with positive bisectional curvature decays at least linearly in the average sense. The third result is concerned with the relation between the volume growth and the curvature decay. We prove that the curvature decay of a complete noncompact Kähler manifold with nonnegative curvature operator and with the maximal volume growth is precisely quadratic in certain average sense.
\end{abstract}

\section{Introduction}

This paper is concerned with the geometric properties of positively curved complete noncompact manifolds near the infinities. Let us first consider the volume growth of the manifolds. When an $m$-dimensional complete noncompact Riemannian manifold has nonnegative Ricci curvature, the classical Bishop volume comparison theorem implies that the volume growth is at most as the Euclidean volume growth. On the other hand, Calabi and Yau (see [25]) showed that the volume growth of a complete noncompact $m$-dimensional Riemannian manifold with nonnegative Ricci curvature must be at least of linear, i.e.,

$$
\operatorname{Vol}\left(B\left(x_{0}, r\right)\right) \geq c r, \quad \text { for all } \quad 1 \leq r<+\infty,
$$

Received August 20, 2004. 
where $\operatorname{Vol}\left(B\left(x_{0}, r\right)\right)$ is the volume of the geodesic ball centered at $x_{0} \in M$ with radius $r$, and $c$ is some positive constant depending on $x_{0}$ and the dimension $m$. The first result of this paper is the following volume growth estimate for Kähler manifolds with nonnegative holomorphic bisectional curvature.

Theorem 1 Let $M$ be a complex n-dimensional complete noncompact Kähler manifold with nonnegative holomorphic bisectional curvature. Suppose also its holomorphic bisectional curvature is positive at least at one point. Then the volume growth of $M$ satisfies

$$
\operatorname{Vol}\left(B\left(x_{0}, r\right)\right) \geq c r^{n}, \quad \text { for all } 1 \leq r<+\infty,
$$

where $c$ is some positive constant depending on $x_{0}$ and the dimension $n$.

Next we study the curvature behavior near the infinities. We still consider complete noncompact Kähler manifolds with positive holomorphic bisectional curvature. In view of the classical Bonnet-Myers theorem, the Ricci curvature can not be uniformly bounded from below by a positive constant. Our second result shows that the curvature actually decays at least linearly in the average sense.

Theorem 2 Let $M$ be a complete noncompact Kähler manifold with positive holomorphic bisectional curvature. Then for any $x_{0} \in M$, there exists a positive constant $C$ such that

$$
\frac{1}{\operatorname{Vol}\left(B\left(x_{0}, r\right)\right)} \int_{B\left(x_{0}, r\right)} R(x) d x \leq \frac{C}{1+r}, \quad \text { for all } 0 \leq r<+\infty,
$$

where $R(x)$ is the scalar curvature of $M$.

The well-known conjecture of Yau on uniformization theorems asks if a complete noncompact Kähler manifold with positive holomorphic bisectional curvature is biholomorphic to a complex Euclidean space. The combination of the above Theorem 2 and the main theorem of [8] gives the following partial affirmative answer to the Yau's conjecture.

Corollary Let $M$ be a complex two-dimensional complete noncompact Kähler manifold with bounded and positive holomorphic bisectional curvature. Suppose the volume growth of $M$ is maximal, i.e.,

$$
\operatorname{Vol}\left(B\left(x_{0}, r\right)\right) \geq c r^{4}, \text { for all } 0 \leq r<+\infty,
$$

for some point $x_{0} \in M$ and some positive constant $c$. Then $M$ is biholomorphic to the complex Euclidean space $\mathbb{C}^{2}$. 
Further we consider the relation between the volume growth and the curvature decay. In [18], Mok-Siu-Yau studied complete Kähler manifold with nonnegative holomorphic bisectional curvature and with the Euclidean volume growth. They showed that the curvatures can not decay faster than quadratically unless the manifolds are flat. It was further shown in [9], [19] this result is still valid without restriction on the volume growth. On the other hand, Yau predicted in [26] that if the volume growth is of Euclidean, then the curvature must decay quadratically in certain average sense. In [8], under an additional assumption that the scalar curvature tends to zero at infinity in the average sense, Tang and the authors confirmed this for the complex surface case by using some special features in dimension 2 such as the Gauss-Bonnet-Chern formula and the classification of holonomy algebras for 4-dimensional Riemannian manifolds. The third result of the present paper is the following affirmative answer to this prediction of Yau for all dimensions under a more restricted curvature assumption.

Theorem 3 Let $M$ be a complex n-dimensional complete noncompact Kähler manifold with bounded and nonnegative curvature operator. Suppose that there exists a positive constant $c_{1}$ such that for a fixed base point $x_{0}$, we have

$$
\text { (*) } \operatorname{Vol}\left(B\left(x_{0}, r\right)\right) \geq c_{1} r^{2 n}, \quad \text { for all } 0 \leq r<+\infty .
$$

Then there exists a constant $c_{2}>0$ such that for all $x \in M, r>0$, we have

$$
\int_{B(x, r)} \frac{R(y)}{d(x, y)^{2 n-2}} d y \leq c_{2} \log (2+r) .
$$

In particular,

$$
\frac{1}{\operatorname{Vol}\left(B\left(x_{0}, r\right)\right)} \int_{B\left(x_{0}, r\right)} R(x) d x \leq c_{3} \frac{\log (2+r)}{r^{2}}, \quad \text { for all } \quad 0 \leq r<+\infty,
$$

for some constant $c_{3}>0$.

There are plenty of complete noncompact convex surfaces in $\mathbb{R}^{3}$ with quadratic volume growth (i.e., cone-like at infinity). All of them are complex 1-dimensional Kähler manifolds satisfying the assumptions of Theorem 3. Clearly any product of finitely many such Kähler manifolds still satisfies the assumptions of Theorem 3. In this way we can produce a lot of complete noncompact Kähler manifolds satisfying all assumptions of Theorem 3 .

On a Kähler manifold, the curvature operator of the Kähler metric reduces to the space $\bigwedge^{1,1}$ of $(1,1)$-forms, this is, the curvature operator $R_{m}: \bigwedge^{1,1} \rightarrow$ $\bigwedge^{1,1}$ and $R_{m} \equiv 0$ on the subspace of $\bigwedge^{2}$ perpendicular to $\bigwedge^{1,1}$. Thus the best possibility for a Kähler metric is the positivity of its curvature operator restricted 
on the subspace $\bigwedge^{1,1}$. In the last part of this paper we will show that the complete noncompact Kähler manifolds, constructed by Cao in [4], have strictly positive curvature operator when restricted on $(1,1)$-forms and satisfy the all assumptions of Theorem 3.

This paper contains seven sections. In Section 2 we give the proof of Theorem 1. Section 3 is devoted to the proof of Theorem 2. In Section 4 we establish some basic estimates to the Ricci flow. In Section 5 we obtain the crucial time decay estimate for the Ricci flow and Theorem 3 will be proved in Section 6 . In the last section we will give a family of complete noncompact Kähler manifolds which have strictly positive curvature operator restricted on $(1,1)$-forms, have the Euclidean volume growth and have quadratic curvature decay.

We are grateful to L.F.Tam for many helpful discussions and Professor S.T.Yau for his encouragement.

\section{The Volume Growth Estimate}

Let us first recall a cut-off function constructed by Schoen and Yau (see Theorem 1.4.2 in $[20])$.

Lemma 2.1 (Schoen-Yau [20]) Suppose $M$ is an m-dimensional complete Riemannian manifold with nonnegative Ricci curvature. Then there exists a constant $C(m)>0$, depending only on the dimension $m$, such that for any $x_{0} \in M$ and any number $0<r<+\infty$, there exists a smooth function $\varphi_{r} \in C^{\infty}(M)$ satisfying

$$
\begin{aligned}
e^{-C(m)\left(1+\frac{d\left(x, x_{0}\right)}{r}\right)} & \leq \varphi_{r}(x) \leq e^{-\left(1+\frac{d\left(x, x_{0}\right)}{r}\right)}, \\
\left|\nabla \varphi_{r}(x)\right| & \leq \frac{C(m)}{r} \varphi_{r}(x), \\
\left|\Delta \varphi_{r}(x)\right| & \leq \frac{C(m)}{r^{2}} \varphi_{r}(x),
\end{aligned}
$$

for $x \in M$, where $d\left(x, x_{0}\right)$ is the distance between $x$ and $x_{0}$.

Proof. In [20], Schoen and Yau constructed a $C^{\infty}$ function $f$ satisfying

$$
\begin{gathered}
\frac{1}{C}\left(1+d\left(x, x_{0}\right)\right) \leq f(x) \leq 1+d\left(x, x_{0}\right), \\
|\nabla f(x)| \leq C,
\end{gathered}
$$




$$
|\Delta f(x)| \leq C,
$$

for all $x \in M$ and some positive constant $C$ depending only on the dimension $m$. Denote by $g_{i j}(x)$ the Riemannian metric of $M$. We define a new metric on $M$ by

$$
\tilde{g}_{i j}(x)=\frac{1}{r^{2}} g_{i j}(x), \quad x \in M .
$$

Clearly the new metric $\tilde{g}_{i j}(x)$ is still a complete Riemannian metric on $M$ with nonnegative Ricci curvature. Thus there exists a smooth function $f_{r}(x) \in C^{\infty}(M)$ such that

$$
\begin{gathered}
\frac{1}{C}\left(1+\frac{d\left(x, x_{0}\right)}{r}\right) \leq f_{r}(x) \leq 1+\frac{d\left(x, x_{0}\right)}{r}, \\
\left|\tilde{\nabla} f_{r}(x)\right|_{\tilde{g}} \leq C, \\
\left|\tilde{\Delta} f_{r}(x)\right|_{\tilde{g}} \leq C,
\end{gathered}
$$

for all $x \in M$. Here $\tilde{\nabla}, \tilde{\Delta}$ and $|\cdot|_{\tilde{g}}$ are the covariant derivative, Laplacian and norm with respect to the new metric $\tilde{g}_{i j}(x)$, and $d\left(x, x_{0}\right)$ is the geodesic distance with respect to the original metric $g_{i j}(x)$. Therefore by setting $\varphi_{r}(x)=\exp \left(-f_{r}(x)\right)$ we get the desired cut-off function.

Let $M$ be a complex $n$-dimensional complete noncompact Kähler manifold with nonnegative holomorphic bisectional curvature. Let $x_{0}$ be an arbitrary point in $M$. Since $M$ is complete and noncompact, there is a geodesic ray, say $\gamma(t)$, emanating from $x_{0}$. The family of functions $\eta_{t}: M \rightarrow \mathbb{R}$ defined by $\eta_{t}(x)=d\left(x_{0}, \gamma(t)\right)-d(x, \gamma(t))$ is Lipschitz continuous (with Lipschitz constant 1) and also satisfies $\left|\eta_{t}(x)\right| \leq d\left(x, x_{0}\right)$ (by the triangle inequality). It is thus an equicontinuous family uniformly bounded on compact sets. By Ascoli-Arzela's theorem, a subsequence of $\left\{\eta_{t}\right\}$ converges to Lipschitz continuous (with Lipschitz constant 1) function $\eta: M \rightarrow \mathbb{R}$, the convergence being uniform on compact subsets of $M$. This function $\eta$ is called the Busemann function of $\gamma$ (Cheeger-Gromoll [6]). It was shown in [24] (see Theorem A(c) of [24]) that $\eta$ is plurisubharmonic and is strictly plurisubharmonic at the points where the bisectional curvature is positive. We now use this plurisubharmonic function to prove Theorem 1.

Proof of Theorem 1. Without loss of generality, we may assume that the holomorphic bisectional curvature is positive at $x_{0}$. Then the Busemann function $\eta$ is strictly plurisubharmonic in a neighborhood of $x_{0}$. Since the holomorphic bisectional curvature is nonnegative, the Ricci curvature is also nonnegative. By Lemma 2.1 we obtain the cut-off function $\varphi_{r}$ for any given $r>0$.

Fix a small positive number $\delta$ and a large positive number $r$. Let $\rho: \mathbb{R}^{2 n} \rightarrow \mathbb{R}$ be a nonnegative smooth function supported in the unit ball centered at the origin 
of $\mathbb{R}^{2 n}$, with

$$
\int_{\mathbb{R}^{2 n}} \rho(v) d v=1
$$

We set

$$
\rho_{\varepsilon}(v)=\frac{1}{\varepsilon^{2 n}} \rho\left(\frac{v}{\varepsilon}\right), \quad \text { for } \quad v \in \mathbb{R}^{2 n}, \varepsilon>0
$$

and

$$
\left(\eta * \rho_{\varepsilon}\right)(x)=\int_{\mathbb{R}^{2 n}} \rho_{\varepsilon}(v) \eta\left(\exp _{x}(v)\right) d v, \quad \text { for } \quad x \in M .
$$

Clearly $\eta * \rho_{\varepsilon}$ is smooth and converges to $\eta$ uniformly on compact sets as $\varepsilon \rightarrow 0$. It is well-known (see for example [10]) that $\eta * \rho_{\varepsilon}$ is also plurisubharmonic on any compact subset as $\varepsilon>0$ small enough. Denote by $\omega$ the Kähler form of $M$. We compute

$$
\begin{aligned}
& \int_{\left\{\varphi_{r}>\delta\right\}}\left(\varphi_{r}-\delta\right)^{n}(\sqrt{-1})^{n}\left(\partial \bar{\partial}\left(\eta * \rho_{\varepsilon}\right)\right)^{n} \\
= & -\int_{\left\{\varphi_{r}>\delta\right\}} n\left(\varphi_{r}-\delta\right)^{n-1}(\sqrt{-1})^{n} \partial \varphi_{r} \wedge \bar{\partial}\left(\eta * \rho_{\varepsilon}\right) \wedge\left(\partial \bar{\partial}\left(\eta * \rho_{\varepsilon}\right)\right)^{n-1} \\
\leq & \int_{\left\{\varphi_{r}>\delta\right\}} \frac{2 n C(2 n)}{r}\left(\varphi_{r}-\delta\right)^{n-1} \varphi_{r}(\sqrt{-1})^{n-1}\left(\partial \bar{\partial}\left(\eta * \rho_{\varepsilon}\right)\right)^{n-1} \wedge \omega \\
\leq & \int_{\left\{\varphi_{r}>\delta\right\}} \frac{(2 n C(2 n))^{2}}{r^{2}}\left(\varphi_{r}-\delta\right)^{n-2} \varphi_{r}^{2}(\sqrt{-1})^{n-2}\left(\partial \bar{\partial}\left(\eta * \rho_{\varepsilon}\right)\right)^{n-2} \wedge \omega^{2} \\
\ldots & \int_{\left\{\varphi_{r}>\delta\right\}} \frac{(2 n C(2 n))^{n}}{r^{n}} \varphi_{r}^{n} \omega^{n} .
\end{aligned}
$$

Since $\eta$ is strictly plurisubharmonic in a neighborhood of $x_{0}$, by letting $\varepsilon \rightarrow 0$ and then $\delta \rightarrow 0$, we know that there is a positive number $c_{0}>0$, depending on $x_{0}$ and the dimension $n$, such that as $r \geq 1$,

$$
\begin{aligned}
0<c_{0} & \leq \int_{M} \varphi_{r}^{n}(\sqrt{-1})^{n}(\partial \bar{\partial} \eta)^{n} \\
& \leq \frac{(2 n C(2 n))^{n}}{r^{n}} \int_{M} \varphi_{r}^{n} \omega^{n} \\
& \leq \frac{(2 n C(2 n))^{n}}{r^{n}} \int_{M} \varphi_{r} \omega^{n} .
\end{aligned}
$$


By using the standard volume comparison and Lemma 2.1, we have

$$
\begin{aligned}
\int_{M} \varphi_{r} \omega^{n} \leq & \int_{B\left(x_{0}, r\right)} e^{-\left(1+\frac{d\left(x, x_{0}\right)}{r}\right)} \omega^{n} \\
& +\sum_{k=0}^{\infty} \int_{B\left(x_{0}, 2^{k+1} r\right) \backslash B\left(x_{0}, 2^{k} r\right)} e^{-\left(1+\frac{d\left(x, x_{0}\right)}{r}\right)} \omega^{n} \\
\leq & \operatorname{Vol}\left(B\left(x_{0}, r\right)\right)+\sum_{k=0}^{\infty} e^{-2^{k}}\left(2^{k+1}\right)^{2 n} \operatorname{Vol}\left(B\left(x_{0}, r\right)\right) \\
\leq & C \operatorname{Vol}\left(B\left(x_{0}, r\right)\right),
\end{aligned}
$$

where $C$ is some positive constant depending only on the dimension $n$.

Thus a combination of (2.2) and (2.3) gives the desired volume growth estimate.

Remark 2.2 Klembeck [15] (see also [23]) and Cao [3] (see Remark 1.3 in [3]) presented some complete Kähler metrics on $\mathbb{C}^{n}$ which have positive holomorphic bisectional curvature everywhere such that the volume of the geodesic ball $B(O, r)$ centered at the origin $O$ with respect to the Kähler metric grows like $r^{n}$. Thus the volume growth estimate of Theorem 1 is sharp.

Remark 2.3 The assumption that the holomorphic bisectional curvature is positive at least at one point is necessary. Indeed, let $M_{1}$ be a noncompact convex surface in $\mathbb{R}^{3}$ which is asymptotic to a cylinder at infinity. Clearly $M_{1}$ is a complete noncompact Riemannian surface with positive curvature and has linear volume growth. Also let $\mathbb{C} P^{n-1}$ be the complex projective space with the Fubini-Study metric. Then the product $M_{1} \times \mathbb{C} P^{n-1}$ is a complex $n$-dimensional complete noncompact Kähler manifold with nonnegative holomorphic bisectional curvature. But its volume growth is of linear.

Remark 2.4 In view of Theorem 1, it is naturally raised a question whether there is a similar volume growth estimate for Riemannian manifold with positive sectional curvature. The answer is negative. More precisely, for each dimension $n \geq 2$, there exist $n$-dimensional Riemannian manifolds which have positive sectional curvature everywhere but have linear volume growth. In fact on any bounded, convex and smooth domain $\Omega$ in $\mathbb{R}^{n}$, we can choose a strictly convex function $u(x)$ defined over $\Omega$ which tends to $+\infty$ as $x$ approaches to the boundary $\partial \Omega$. The graph of the convex function $u(x)$ is a hypersurface in $\mathbb{R}^{n+1}$, denoted by $M^{n}$. Clearly the hypersurface $M^{n}$ is strictly convex, i.e., the second fundamental form is strictly positive definite. It then follows from the Gauss equation that $M^{n}$ has strictly positive sectional curvature. Since the domain $\Omega$ is bounded, the 
volume growth of $M^{n}$ must be of linear.

\section{The Minimal Curvature Decay}

In this section, we will prove that the curvature of complete positive curved Kähler manifold decays at least linearly.

Proof of Theorem 2. Let $\eta(x)$ be a Busemann function constructed from a ray initiating from $x_{0}$. By a smoothing argument, we may assume $\eta(x)$ is smooth, $|d \eta(x)| \leq 2$, and the Levi form $\sqrt{-1} \partial \bar{\partial} \eta(x)$ of $\eta(x)$ is strictly positive on $M$.

Let $K_{M}$ be the canonical line bundle of $M$, then there exists a continuous positive function $\lambda(x)$ on $M$, such that for any $k>0$,

$$
\sqrt{-1} \partial \bar{\partial}(k \eta(x))+C\left(K_{M}\right)+R i c \geq k \lambda(x) \omega(x)
$$

where $C\left(K_{M}\right)$ be the curvature of $K_{M}$, and $\omega(x)$ is the Kähler form of $M$.

By the $L^{2}$-estimates of $\bar{\partial}$-operator of Hörmander $([14])$, there exist a positive constant $k_{0}>0$, and a nontrivial holomorphic section $S$ of $K_{M}$ such that

$$
\int_{M}\|S\|^{2} e^{-k_{0} \eta(x)} d V(x)<+\infty .
$$

and $\left\|S\left(x_{0}\right)\right\|^{2}=1$.

Recall the Poincaré-Lelong equation

$$
\sqrt{-1} \partial \bar{\partial} \log \|S\|^{2}=[S=0]+R i c
$$

in the sense of currents. And for any $0<\delta<1$, a direct computation gives

$$
\Delta \log \left(\|S\|^{2}+\delta\right) \geq R(x) \frac{\|S\|^{2}}{\|S\|^{2}+\delta}
$$

and

$$
\Delta\|S\|^{2} \geq 0,
$$

in the classical sense. By the mean-value-inequality (see [17]), we have for any $x \in M, r>0$,

$$
\|S\|^{2}(x) \leq \frac{c(n)}{\operatorname{Vol}(B(x, r))} \int_{B(x, r)}\|S\|^{2} .
$$


Here and in the followings, $c(n)$ is denoted by various positive constant depending only on the dimension.

Choosing $r=d\left(x, x_{0}\right)+1$ and noting that $\eta(x) \leq 2\left(d\left(x, x_{0}\right)+1\right)$, we have

$$
\begin{aligned}
\|S\|^{2}(x) & \leq \frac{c(n)}{\operatorname{Vol}\left(B\left(x_{0}, 1\right)\right)} \int_{B\left(x_{0}, 1+d\left(x, x_{0}\right)\right)}\|S\|^{2} \\
& \leq \frac{c(n) e^{2 k_{0} d\left(x, x_{0}\right)}}{\operatorname{Vol}\left(B\left(x_{0}, 1\right)\right)} \int_{M}\|S\|^{2} e^{-k_{0} \eta} \\
& \leq \text { const. } e^{2 k_{0} d\left(x, x_{0}\right)}
\end{aligned}
$$

hence

$$
\log \|S\|^{2}(x) \leq c_{1} d\left(x, x_{0}\right)+c_{2}, \quad \text { for some } c_{1}, c_{2}>0
$$

Suppose $M$ admits a positive Green function $G=G\left(x, x_{0}\right)$. Then for any $\alpha>0, \beta>0, \varepsilon>0$ and $0<\delta<1$, it follows from (3.3) that

$$
\begin{aligned}
& \int_{\{\beta>G>\alpha\}} R(x) \frac{\|S\|^{2}(x)}{\|S\|^{2}(x)+\delta}\left(G\left(x, x_{0}\right)-\alpha\right)^{1+\varepsilon} \\
\leq & \int_{\{\beta>G>\alpha\}} \Delta \log \left(\|S\|^{2}+\delta\right)(G-\alpha)^{1+\varepsilon} \\
= & \int_{\{\beta>G>\alpha\}} \log \left(\|S\|^{2}+\delta\right) \Delta(G-\alpha)^{1+\varepsilon} \\
& +\int_{\{G=\beta\}} \frac{\partial \log \left(\|S\|^{2}+\delta\right)}{\partial \vec{n}}(G-\alpha)^{1+\varepsilon} \\
& -(1+\varepsilon) \int_{\{G=\beta\}} \log \left(\|S\|^{2}+\delta\right)(G-\alpha)^{\varepsilon} \frac{\partial G}{\partial \vec{n}}
\end{aligned}
$$

where $\vec{n}$ is the outer normal vector of $\partial\{\beta>G>\alpha\}$.

Since

$$
\Delta(G-\alpha)^{1+\varepsilon}=(1+\varepsilon)(G-\alpha)^{\varepsilon-1}|\nabla G|^{2} \geq 0,
$$

on $\{\beta>G>\alpha\}$, we get

$$
\int_{\{\beta>G>\alpha\}} \log \left(\|S\|^{2}+\delta\right) \Delta(G-\alpha)^{1+\varepsilon}
$$




$$
\begin{aligned}
& \leq \sup _{\{\beta>G>\alpha\}} \log \left(\|S\|^{2}+\delta\right) \int_{\{\beta>G>\alpha\}} \Delta(G-\alpha)^{1+\varepsilon} \\
& =\sup _{\{\beta>G>\alpha\}} \log \left(\|S\|^{2}+\delta\right) \int_{\{G=\beta\}}(1+\varepsilon)(G-\alpha)^{\varepsilon} \frac{\partial G}{\partial \vec{n}} .
\end{aligned}
$$

Letting $\varepsilon \rightarrow 0$, it follows

$$
\begin{aligned}
\int_{\{\beta>G>\alpha\}} R(x) \frac{\|S\|^{2}(x)}{\|S\|^{2}(x)+\delta}\left(G\left(x, x_{0}\right)-\alpha\right) \leq & \sup _{\{G>\alpha\}} \log \left(\|S\|^{2}+\delta\right) \int_{\{G=\beta\}} \frac{\partial G}{\partial \vec{n}} \\
& +\int_{\{G=\beta\}}(G-\alpha) \frac{\partial \log \left(\|S\|^{2}+\delta\right)}{\partial \vec{n}} \\
& -\int_{\{G=\beta\}} \frac{\partial G}{\partial \vec{n}} \log \left(\|S\|^{2}+\delta\right) .
\end{aligned}
$$

On $\{G=\beta\}, G$ and $\frac{\partial G}{\partial \vec{n}}$ are asymptotic to $\frac{c(n)}{d^{2 n-2}}$ and $(2 n-2) c(n) \frac{1}{d^{2 n-1}}$ respectively, as $\beta \rightarrow+\infty$. So we have

$$
\left|\int_{\{G=\beta\}}(G-\alpha)\right| \leq c(n) \frac{d^{2 n-1}}{d^{2 n-2}}=c(n) d \rightarrow 0, \text { as } \beta \rightarrow+\infty,
$$

and

$$
\int_{\{G=\beta\}} \frac{\partial G}{\partial \vec{n}} \rightarrow c(n), \text { as } \beta \rightarrow+\infty .
$$

Hence, letting $\beta \rightarrow+\infty$ in (3.6), it follows

$$
\int_{\{G>\alpha\}} R(x) \frac{\|S\|^{2}(x)}{\|S\|^{2}(x)+\delta}\left(G\left(x, x_{0}\right)-\alpha\right) \leq c(n) \sup _{\{G>\alpha\}} \log \left(\|S\|^{2}+\delta\right)
$$

since $\|S\|\left(x_{0}\right)=1$. This implies

$$
\int_{\{G>2 \alpha\}} R(x) \frac{\|S\|^{2}(x)}{\|S\|^{2}(x)+\delta} G\left(x, x_{0}\right) \leq c(n) \sup _{\{G>\alpha\}} \log \left(\|S\|^{2}+\delta\right) .
$$

Then letting $\delta \rightarrow 0$, we obtain,

$$
\int_{\{G>2 \alpha\}} R(x) G\left(x, x_{0}\right) \leq c(n) \sup _{\{G>\alpha\}} \log \|S\|^{2} .
$$

Since the positive Green's function may not exist on $M$ in general, we consider $\tilde{M}=M \times \mathbb{C}^{2}$, equipped with the product metric. By regarding $\|S\|^{2}$ as a function 
on $\tilde{M}$, the inequality (3.3) still holds. Let $z_{0}=\left(x_{0}, 0\right), z=(x, y) \in \tilde{M}$, the minimal positive Green function of $\tilde{M}$ exists and satisfies

$$
\frac{c(n)^{-1} \tilde{d}^{2}\left(z, z_{0}\right)}{\operatorname{Vol}\left(\tilde{B}\left(z_{0}, \tilde{d}\left(z, z_{0}\right)\right)\right.} \leq \tilde{G}\left(z, z_{0}\right) \leq \frac{c(n) \tilde{d}^{2}\left(z, z_{0}\right)}{\operatorname{Vol}\left(\tilde{B}\left(z_{0}, \tilde{d}\left(z, z_{0}\right)\right)\right.},
$$

where $\tilde{d}^{2}\left(z, z_{0}\right)=d^{2}\left(x, x_{0}\right)+|y|^{2}$.

In fact, (3.8) comes from the following well-known estimate ([20]):

$$
c(n)^{-1} \int_{\tilde{d}^{2}\left(z, z_{0}\right)}^{\infty} \frac{d t}{\operatorname{Vol}\left(\left(\tilde{B}\left(z_{0}, \sqrt{t}\right)\right)\right.} \leq \tilde{G}\left(z, z_{0}\right) \leq c(n) \int_{\tilde{d}^{2}\left(z, z_{0}\right)}^{\infty} \frac{d t}{\operatorname{Vol}\left(\tilde{B}\left(z_{0}, \sqrt{t}\right)\right)},
$$

in particular, we have

$$
\tilde{G}\left(z, z_{0}\right) \geq \frac{c(n)^{-1} a^{2}}{\operatorname{Vol}\left(\tilde{B}\left(z_{0}, a\right)\right)}, \quad \text { for all } z \in \tilde{B}\left(z_{0}, a\right) .
$$

The same argument of obtaining (3.7) gives us

$$
\int_{\{\tilde{G}>2 \alpha\}} R(x) \tilde{G}\left(z, z_{0}\right) \leq c(n) \sup _{\{\tilde{G}>\alpha\}} \log \|S\|^{2} .
$$

For $\alpha>0$, let $r(\alpha)$ be the maximum positive number such that

$$
\tilde{B}\left(z_{0}, r(\alpha)\right) \subset\{\tilde{G}>\alpha\}
$$

which, together with (3.8), implies

$$
\frac{c(n)^{-1} r^{2}(\alpha)}{\operatorname{Vol}\left(\tilde{B}\left(z_{0}, r(\alpha)\right)\right)}<\alpha<\frac{c(n) r^{2}(\alpha)}{\operatorname{Vol}\left(\tilde{B}\left(z_{0}, r(\alpha)\right)\right)} .
$$

Since

$$
B\left(x_{0}, \frac{1}{2} r(\alpha)\right) \times B\left(0, \frac{1}{2} r(\alpha)\right) \subset \tilde{B}\left(z_{0}, r(\alpha)\right) \subset B\left(x_{0}, r(\alpha)\right) \times B(0, r(\alpha)),
$$

we get by combining with (3.8) that for any $z$ with $G\left(z, z_{0}\right)>\alpha$,

$$
\frac{c(n)^{-1} r^{2}(\alpha)}{\operatorname{Vol}\left(\tilde{B}\left(z_{0}, r(\alpha)\right)\right)} \leq \frac{\tilde{d}^{2}\left(z, z_{0}\right)}{\operatorname{Vol}\left(\tilde{B}\left(z_{0}, \tilde{d}\left(z, z_{0}\right)\right)\right)}
$$

i.e.,

$$
\frac{\operatorname{Vol}\left(\tilde{B}\left(z_{0}, \tilde{d}\left(z, z_{0}\right)\right)\right)}{\operatorname{Vol}\left(\tilde{B}\left(z_{0}, r(\alpha)\right)\right)} \leq c(n) \frac{\tilde{d}^{2}\left(z, z_{0}\right)}{r^{2}(\alpha)}
$$


and then

$$
\frac{\operatorname{Vol}\left(B\left(x_{0}, \frac{1}{2} \tilde{d}\left(z, z_{0}\right)\right)\right)}{\operatorname{Vol}\left(B\left(x_{0}, r(\alpha)\right)\right)} \leq c(n) \frac{r^{2}(\alpha)}{\tilde{d}^{2}\left(z, z_{0}\right)} .
$$

Thus if $\tilde{d}\left(z, z_{0}\right) \geq 2 r(\alpha)$, we get that $r(\alpha) \geq c^{-1}(n) d\left(z, z_{0}\right)$. So we get

$$
\tilde{B}\left(z_{0}, r(\alpha)\right) \subset\{\tilde{G}>\alpha\} \subset \tilde{B}\left(z_{0}, c(n) r(\alpha)\right) .
$$

By (3.9), (3.10), (3.11) and (3.12), we have

$$
\frac{r^{2}(\alpha)}{\operatorname{Vol}\left(B\left(x_{0}, r(\alpha)\right)\right)} \int_{B\left(x_{0}, r(\alpha)\right)} R(x) d V(x) \leq c(n) \sup _{B\left(x_{0}, c(n) r(\alpha)\right)} \log \|S\|^{2} .
$$

Combining (3.5), we get for any $a>0$,

$$
\frac{1}{\operatorname{Vol}\left(B\left(x_{0}, a\right)\right)} \int_{B\left(x_{0}, a\right)} R(x) d V(x) \leq \frac{C}{a+1} .
$$

Therefore the proof of the theorem is completed.

\section{The Ricci Flow and Preliminary Estimates}

From now on we consider $\left(M, g_{\alpha \bar{\beta}}\right)$ to be a complete noncompact Kähler manifold satisfying the assumptions of Theorem 3. Our method to prove Theorem 3 is not directly working on the Kähler metric. Instead we use the Kähler metric as initial data and evolve it by Hamiltion's Ricci flow. We study the dynamic property of the evolving metric and prove that the curvature of the evolving metric decays linearly in time. The curvature of the evolving metric satisfies a nonlinear heat equation. Intuitively, the Harnack inequality of heat equation bridges the time decay with the space decay. By using the time decay estimate on the evolving curvature we will be indeed able to prove that the curvature of the initial metric decays quadratically in space in average sense. In this section we give some preliminary estimates for the Ricci flow.

Let us evolve the metric $g_{\alpha \bar{\beta}}$ according to the following Ricci flow equation

$$
\left\{\begin{aligned}
\frac{\partial g_{\alpha \bar{\beta}}}{\partial t}(x, t) & =-R_{\alpha \bar{\beta}}(x, t), \quad x \in M, t>0 \\
g_{\alpha \bar{\beta}}(x, 0) & =g_{\alpha \bar{\beta}}(x), \quad x \in M
\end{aligned}\right.
$$

where $R_{\alpha \bar{\beta}}(x, t)$ denotes the Ricci curvature tensor of the metric $g_{\alpha \bar{\beta}}(x, t)$. 
It was shown in [21] that the Ricci flow (4.1) has a maximal solution $g_{\alpha \bar{\beta}}(x, t)$ on $M \times\left[0, T_{\max }\right)$ with either $T_{\max }=+\infty$ or $0<T_{\max }<+\infty$ and the curvature becomes unbounded as $t \rightarrow T_{\max }(<+\infty)$. Since the initial Kähler metric has nonnegative curvature operator, it is known from [11], [22] that the nonnegativity of the curvature operator and Kählerity are preserved under the evolution of (4.1).

When the initial metric satisfies an additional assumption that the initial curvature decays pointwisely to zero at infinity (i.e., $\lim _{d\left(x, x_{0}\right) \rightarrow+\infty} R(x)=0$ ), one knows from Hamilton [13] (Theorem 18.3 in [13]) that the Euclidean volume growth condition is preserve under the Ricci flow. Thanks to Theorem 2, we can remove the curvature decay assumption in the following result.

Lemma 4.1 Suppose $\left(M, g_{\alpha \bar{\beta}}\right)$ is a complex $n$-dimensional complete noncompact simply connected Kähler manifold with bounded and nonnegative curvature operator and satisfies the conditions (*) in Theorem 3. Then the Euclidean volume growth condition (*) is preserved under the evolution of (4.1), i.e.,

$$
\operatorname{Vol}_{t}\left(B_{t}(x, r)\right) \geq c_{1} r^{2 n}, \quad \text { for all } \quad r>0, x \in M \quad \text { and } t \in\left[0, T_{\max }\right),
$$

with the same constant $c_{1}$ in the condition $\left(^{*}\right)$. Here $B_{t}(x, r)$ is the geodesic ball of radius $r$ centered at $x$ with respect to the metric $g_{\alpha \bar{\beta}}(\cdot, t)$, and the volume $V_{\text {ol }}$ is taken with respect to the metric $g_{\alpha \bar{\beta}}(\cdot, t)$.

Proof. Let us first consider the evolution equation of the curvature operator of the evolving metric $g_{\alpha \bar{\beta}}(x, t)$. By applying the strong maximum principle to the evolution equation, it was shown by Hamilton [11] (see Theorem 8.3 of [11]) that there exists a small positive constant $\delta_{0}\left(<T_{\max }\right)$ such that on the time interval $0<t<\delta_{0}$, the image of the curvature operator of $g_{\alpha \bar{\beta}}(\cdot, t)$ is invariant under parallel translation and constant in time. Since the curvature of $g_{\alpha \bar{\beta}}(\cdot, \delta)$ is uniformly bounded for $\delta \in\left[0, \delta_{0}\right]$, it is easy to see from the evolution equation (4.1) that each metric $g_{\alpha \bar{\beta}}(\cdot, \delta)$ is quasi-isometric to the initial metric $g_{\alpha \bar{\beta}}(\cdot)$ and satisfies

$$
\operatorname{Vol}_{\delta}\left(B_{\delta}\left(x_{0}, r\right)\right) \geq c_{1}(\delta) r^{2 n}, \quad \text { for all } 0 \leq r<+\infty, \delta \in\left[0, \delta_{0}\right],
$$

where the function $c_{1}(\delta)$ is defined and positive on $\left[0, \delta_{0}\right]$ such that $\lim _{\delta \rightarrow 0} c_{1}(\delta)=c_{1}$. Thus, without loss of generality, we may assume that the image of the curvature operator of the initial metric $g_{\alpha \bar{\beta}}$ is invariant under parallel translation. According to the decomposition theorem of de Rham (see for example Theorem 8.1 in [16]), the simply connected, complete Kähler manifold with the metric $g_{\alpha \bar{\beta}}$ is holomorphically isometric to the direct product $\mathbb{C}^{k} \times M_{1} \times \cdots \times M_{m}$, where 
$M_{1}, \cdots, M_{m}$ are all simply connected, complete, irreducible Kähler manifold. Since $M$ has nonnegative curvature operator and satisfies the maximal volume growth condition $(*)$, each $M_{i}, i=1, \cdots, m$, also has nonnegative curvature operator and is noncompact. By applying the well-known theorem of Berger[1], each irreducible Kähler manifold $M_{i}, i=1, \cdots, m$, is either a Hermitian symmetric space or has its holonomy group as $U\left(n_{i}\right), S U\left(n_{i}\right)$ or $S_{p}\left(\frac{n_{i}}{2}\right)$, where $n_{i}$ is the complex dimensional of $M_{i}$. Since $M_{i}$ has nonnegative curvature operator, $M_{i}$ is thus either Ricci flat or has $U\left(n_{i}\right)$ holonomy (see for example [5]). Hence each $M_{i}, i=1, \cdots, m$ has $U\left(n_{i}\right)$ holonomy. By noting that $M_{i}$ is simply connected, the restricted holonomy group agrees with the full holonomy group. Also note that the image of the curvature operator is invariant under parallel translation. We then have from Ambrose-Singer holonomy theorem that the image of the curvature operator at any point $x$ is $u\left(n_{i}\right) \cong \bigwedge_{\mathbb{R}}^{1,1}\left(T_{x} M_{i}\right)$, the space of real $(1,1)$ forms on the tangent space $T_{x} M_{i}$. Therefore, together with the nonnegativity of the curvature operator, it follows that the curvature operator of $M_{i}$ is strictly positive when restricted to $\bigwedge_{\mathbb{R}}^{1,1}\left(T_{x} M_{i}\right)$. In particular the holomorphic bisectional curvature of $M_{i}$ is positive everywhere.

Denote by $R_{i}(x)$ the scalar curvature of the Kähler manifold $M_{i}, i=1, \cdots, m$. We then know from Theorem 2 that the scalar curvature $R_{i}(x)$ decays at least linearly in the average sense. Since the scalar curvature $R(x)$ of $M$ is given by $R_{1}(x)+\cdots+R_{m}(x)$, it follows that

$$
\lim _{r \rightarrow \infty} \frac{1}{\operatorname{Vol}\left(B\left(x_{0}, r\right)\right)} \int_{B\left(x_{0}, r\right)} R(x) d x=0,
$$

where $\operatorname{Vol}\left(B\left(x_{0}, r\right)\right)$ is the volume of the geodesic ball $B\left(x_{0}, r\right)$ with respect to the initial metric $g_{\alpha \bar{\beta}}$.

We have seen that the curvature operator of the evolving metric $g_{\alpha \bar{\beta}}(\cdot, t)$ is nonnegative, and then the Ricci curvature $R_{\alpha \bar{\beta}}(x, t)$ is also nonnegative on $M \times\left[0, T_{\max }\right)$. The equation in (4.1) thus implies that the metric is shrinking in time. In particular,

$$
g_{\alpha \bar{\beta}}(x, t) \leq g_{\alpha \bar{\beta}}(x, 0), \quad \text { on } \quad M \times\left[0, T_{\max }\right) .
$$

Set

$$
F(x, t)=\log \frac{\operatorname{det}\left(g_{\alpha \bar{\beta}}(x, t)\right)}{\operatorname{det}\left(g_{\alpha \bar{\beta}}(x, 0)\right)}, \quad \text { on } \quad M \times\left[0, T_{\max }\right) \text {. }
$$


We then have

$$
\begin{aligned}
e^{F(x, t)} R(x, t) & =g^{\alpha \bar{\beta}}(x, t) R_{\alpha \bar{\beta}}(x, t) \cdot \frac{\operatorname{det}\left(g_{\alpha \bar{\beta}}(x, t)\right)}{\operatorname{det}\left(g_{\alpha \bar{\beta}}(x, 0)\right)} \\
& \leq g^{\alpha \bar{\beta}}(x, 0) R_{\alpha \bar{\beta}}(x, t) \\
& =g^{\alpha \bar{\beta}}(x, 0)\left(R_{\alpha \bar{\beta}}(x, t)-R_{\alpha \bar{\beta}}(x, 0)\right)+R(x, 0) \\
& =-\Delta_{0} F(x, t)+R(x, 0)
\end{aligned}
$$

where $\Delta_{0}$ denotes the Laplacian operator with respect to the initial metric $g_{\alpha \bar{\beta}}(x, 0)$ and $R(x, t)$ denotes the scalar curvature of the metric $g_{\alpha \bar{\beta}}(x, t)$. From the equation in (4.1), we see

$$
\begin{aligned}
\frac{\partial F(x, t)}{\partial t} & =g^{\alpha \bar{\beta}}(x, t) \frac{\partial}{\partial t} g_{\alpha \bar{\beta}}(x, t) \\
& =-R(x, t) .
\end{aligned}
$$

A combination of (4.3) and (4.4) gives

$$
e^{F(x, t)} \frac{\partial F(x, t)}{\partial t} \geq \Delta_{0} F(x, t)-R(x, 0), \quad \text { on } \quad M \times\left[0, T_{\max }\right) .
$$

For any fixed point $x_{0} \in M$ and any number $0<r<+\infty$, we let $\varphi_{r}(x)$ be the cut-off function on $\left(M, g_{\alpha \bar{\beta}}\right)$ obtained in Lemma 2.1. We compute

$$
\begin{aligned}
\frac{\partial}{\partial t} \int_{M} \varphi_{r}(x) e^{F(x, t)} d V_{0} & \geq \int_{M} \varphi_{r}(x)\left(\Delta_{0} F(x, t)-R(x .0)\right) d V_{0} \\
& \geq \frac{C(2 n)}{r^{2}} \int_{M} \varphi_{r}(x) F(x, t) d V_{0}-\int_{M} \varphi_{r}(x) R(x, 0) d V_{0}
\end{aligned}
$$

where $d V_{0}$ denotes the volume element of the initial metric $g_{\alpha \bar{\beta}}$ and we note that $F(x, t)$ is nonpositive. Note from $(4.4)$ that $F(\cdot, t)$ is nonincreasing in time and $F(\cdot, 0) \equiv 0$. We integrate the above inequality from 0 to $t$ to get

$$
\begin{gathered}
\int_{M} \varphi_{r}(x)\left(1-e^{F(x, t)}\right) d V_{0} \leq \frac{C(2 n) t}{r^{2}} \int_{M} \varphi_{r}(x)(-F(x, t)) d V_{0} \\
+t \int_{M} \varphi_{r}(x) R(x, 0) d V_{0} .
\end{gathered}
$$

Since the metric is shrinking under the Ricci flow, we have

$$
B_{t}\left(x_{0}, r\right) \supset B_{0}\left(x_{0}, r\right), \quad \text { for } t \geq 0,0<r<+\infty,
$$


and

$$
\begin{aligned}
\operatorname{Vol}_{t}\left(B_{t}\left(x_{0}, r\right)\right) & \geq \operatorname{Vol}_{t}\left(B_{0}\left(x_{0}, r\right)\right) \\
& =\int_{B_{0}\left(x_{0}, r\right)} e^{F(x, t)} d V_{0} \\
& =\operatorname{Vol}_{0}\left(B_{0}\left(x_{0}, r\right)\right)+\int_{B_{0}\left(x_{0}, r\right)}\left(e^{F(x, t)}-1\right) d V_{0} .
\end{aligned}
$$

By Lemma 2.1 and (4.6), the error term in (4.7) satisfies

$$
\begin{aligned}
\int_{B\left(x_{0}, r\right)}\left(e^{F(x, t)}-1\right) d V_{0} \geq & e^{2 C(2 n)} \int_{M} \varphi_{r}(x)\left(e^{F(x, t)}-1\right) d V_{0} \\
\geq & \frac{C(2 n) e^{2 C(2 n)} t}{r^{2}} \int_{M} \varphi_{r}(x) F(x, t) d V_{0} \\
& -e^{2 C(2 n)} t \int_{M} \varphi_{r}(x) R(x, 0) d V_{0} .
\end{aligned}
$$

Consider any fixed $T_{0}<T_{\max }$. Since the curvature in uniformly bounded on $M \times\left[0, T_{0}\right]$, it is clear from (4.4) that $F(x, t)$ is uniformly bounded on $M \times\left[0, T_{0}\right]$. We then set

$$
A=\sup \left\{|F(x, t)| \quad \mid \quad x \in M, t \in\left[0, T_{0}\right]\right\}
$$

and

$$
\varepsilon(r)=\sup \left\{\frac{1}{V o l_{0}\left(B_{0}\left(x_{0}, a\right)\right)} \int_{B_{0}\left(x_{0}, a\right)} R(x, 0) d V_{0} \quad \mid \quad a \geq r\right\} .
$$

From (4.2) we see that $\varepsilon(r) \rightarrow 0$ as $r \rightarrow+\infty$. By using the volume comparison theorem, we showed in (2.3) that

$$
\int_{M} \varphi_{r}(x) d V_{0} \leq C \operatorname{Vol}_{0}\left(B\left(x_{0}, r\right)\right),
$$

and similarly

$$
\begin{aligned}
\int_{M} \varphi_{r}(x) R(x, 0) d V_{0} \leq & \int_{M} R(x, 0) e^{-\left(1+\frac{d_{0}\left(x_{0}, x\right)}{r}\right)} d V_{0} \\
\leq & \int_{B_{0}\left(x_{0}, r\right)} R(x, 0) d V_{0}+\sum_{k=0}^{\infty} e^{-2^{k}}\left(2^{k+1}\right)^{2 n} \\
& \frac{V o l_{0}\left(B_{0}\left(x_{0}, r\right)\right)}{V o l_{0}\left(B_{0}\left(x_{0}, 2^{k+1} r\right)\right)} \int_{B_{0}\left(x_{0}, 2^{k+1} r\right)} R(x, 0) d V_{0} \\
\leq & C \cdot \varepsilon(r) \cdot \operatorname{Vol}_{0}\left(B_{0}\left(x_{0}, r\right)\right),
\end{aligned}
$$


where $d_{0}\left(x_{0}, x\right)$ is the distance between $x_{0}$ and $x$ with respect to the initial metric $g_{\alpha \bar{\beta}}(\cdot, 0)$ and $C$ is some positive constant depending only on the dimension $n$.

Substituting (4.8),(4.9) and (4.10) into (4.7) and dividing by $r^{2 n}$, we obtain

$$
\begin{aligned}
\frac{V o l_{t}\left(B_{t}\left(x_{0}, r\right)\right)}{r^{2 n}} \geq & \frac{V o l_{0}\left(B_{0}\left(x_{0}, r\right)\right)}{r^{2 n}}-\frac{C(2 n) e^{2 C(2 n)} A T_{0}}{r^{2}}\left(C \frac{V o l_{0}\left(B_{0}\left(x_{0}, r\right)\right)}{r^{2 n}}\right) \\
& -e^{2 C(2 n)} T_{0}\left(C \cdot \varepsilon(r) \cdot \frac{V o l_{0}\left(B_{0}\left(x_{0}, r\right)\right)}{r^{2 n}}\right) \\
= & \left(1-\frac{C(2 n) e^{2 C(2 n)} A T_{0}}{r^{2}}-C e^{2 C(2 n)} T_{0} \varepsilon(r)\right) \frac{V o l_{0}\left(B_{0}\left(x_{0}, r\right)\right)}{r^{2 n}} .
\end{aligned}
$$

Then by letting $r \rightarrow+\infty$, we deduce that

$$
\lim _{r \rightarrow+\infty} \frac{\operatorname{Vol}_{t}\left(B_{t}\left(x_{0}, r\right)\right)}{r^{2 n}} \geq c_{1} .
$$

Hence by using the standard volume comparison we get

$$
\operatorname{Vol}_{t}\left(B_{t}(x, r)\right) \geq c_{1} r^{2 n}, \quad \text { for all } \quad x \in M, 0 \leq r<+\infty \quad \text { and } \quad t \in\left[0, T_{0}\right] .
$$

Finally, since $T_{0}\left(<T_{\max }\right)$ is arbitrary, this completes the proof of the lemma. \#

In the next section we will use rescaling arguments to analyse the behavior of the evolving metric near the maximal time $T_{\max }$. In view of the compactness theorem of Hamilton [12], we need to estimate the injectivity radius of $\left(M, g_{\alpha \bar{\beta}}(\cdot, t)\right)$ in terms of the maximum of the curvature.

Let us recall the local injectivity radius estimate of Cheeger, Gromov and Taylor [7] which says that for any complete Riemannian manifold $N$ of dimension $m$ with $\lambda \leq$ sectional curvature of $N \leq \Lambda$ and let $r$ be a positive constant satisfying $r \leq \pi / 4 \sqrt{\Lambda}$ if $\Lambda>0$, then the injectivity radius of $N$ at a point $x$ is bounded from below as follows

$$
\operatorname{inj}(x, N) \geq r \frac{\operatorname{Vol}(B(x, r))}{\operatorname{Vol}(B(x, r))+V_{\lambda}^{m}(2 r)},
$$

where $V_{\lambda}^{m}(2 r)$ denotes the volume of a ball with radius $2 r$ in the $m$-dimensi- onal space form $V_{\lambda}^{m}$ with constant sectional curvature $\lambda$.

Denote by

$$
R_{\max }(t)=\sup \{R(x, t) \mid x \in M\}, \quad \text { for } \quad t \in\left[0, T_{\max }\right) .
$$


By applying the local injectivity estimate to the evolving manifold $\left(M, g_{\alpha \bar{\beta}}(\cdot, t)\right)$, we get

$$
\begin{aligned}
\operatorname{inj}\left(M, g_{\alpha \bar{\beta}}(\cdot, t)\right) & \geq \frac{\pi}{4 \sqrt{R_{\max }(t)}} \frac{\operatorname{Vol}_{t}\left(B_{t}\left(x, \frac{\pi}{4 \sqrt{R_{\max }(t)}}\right)\right)}{\operatorname{Vol}_{t}\left(B_{t}\left(x, \frac{\pi}{4 \sqrt{R_{\max }(t)}}\right)\right)+V_{0}^{2 n}(1)\left(\frac{\pi}{4 \sqrt{R_{\max }(t)}}\right)^{2 n}} \\
& \geq \frac{\pi}{4 \sqrt{R_{\max }(t)}}\left(\frac{c_{1}}{c_{1}+V_{0}^{2 n}(1)}\right),
\end{aligned}
$$

here we used Lemma 4.1. Thus we have proved

Lemma 4.2 Suppose $\left(M, g_{\alpha \bar{\beta}}\right)$ is assumed in Theorem 3 and let $g_{\alpha \bar{\beta}}(x, t),(x, t) \in$ $M \times\left[0, T_{\max }\right)$, be the maximal solution of (4.1). And suppose $M$ is simply connected. Then there exists a positive constant $\beta$ such that

$$
\operatorname{inj}\left(M, g_{\alpha \bar{\beta}}(\cdot, t)\right) \geq \frac{\beta}{\sqrt{R_{\max }(t)}},
$$

for $t \in\left[0, T_{\max }\right)$.

\section{Curvature Decay in Time}

In this section we analyse the behavior of the evolving metric near the maximal time $T_{\max }$.

Theorem 5.1 Let $\left(M, g_{\alpha \bar{\beta}}(x)\right)$ be a complex $n$-dimensional complete noncompact Kähler manifold satisfying the assumptions of Theorem 3 and let $\left(M, g_{\alpha \bar{\beta}}(x, t)\right)$, $t \in\left[0, T_{\max }\right)$, be the maximal solution of the Ricci flow (4.1) with $g_{\alpha \bar{\beta}}(x)$ as initial metric. Then $T_{\max }=+\infty$ and the scalar curvature $R(x, t)$ of the solution satisfies

$$
0 \leq R(x, t) \leq \frac{C}{1+t}, \quad \text { on } \quad M \times[0,+\infty)
$$

for some positive constant $C$.

Proof. In [8] we established this result for complex 2-dimensional case under an additional assumption that the initial curvature decays to zero at infinity, where we used some special features of complex 2-dimension such as the classification of holonomy algebras and the splitting results in the (real)4-dimensional Riemannian manifolds obtained by the combination of Berger [1] and Hamilton [11]. 
In the followings we don't assume the curvature decay and design a dimension reduction procedure.

According to Hamilton [13], the maximal solution of (4.1) is of either one of the following types.

Type I: $\quad T_{\max }<+\infty$ and $\sup \left(T_{\max }-t\right) R_{\max }(t)<+\infty$;

Type II: $\quad$ either $T_{\max }<+\infty$ and $\sup \left(T_{\max }-t\right) R_{\max }(t)=+\infty$,

or $T_{\max }=+\infty$ and $\sup t R_{\max }(t)=+\infty$;

Type III: $\quad T_{\max }=+\infty$ and $\sup t R_{\max }(t)<+\infty$.

We need to show that the maximal solution must be of Type III. Let us argue by contradiction. Suppose the maximal solution is of Type I or Type II. We first note that we may assume $M$ is simply connected. Indeed by considering the universal covering of $M$, the induced metric of $g_{\alpha \bar{\beta}}(x, t)$ on the universal covering is clearly still a solution to the Ricci flow and satisfies all assumptions of Theorem 3, and of course is still of Type I or Type II.In the previous section we have obtained an injectivity radius estimate for the solution. By combining with a result of Hamilton (Theorem 16.4 and 16.5 in [13]), we know that there exists a sequence of dilations of the solution converging to a limit $\left(\tilde{M}^{(1)}, \tilde{g}_{\alpha \bar{\beta}}^{(1)}(x, t)\right)$ which is a complete solution of the Ricci flow with nonnegative curvature operator, exists for $-\infty<t<\Omega$ for some $0<\Omega \leq+\infty$ and

$$
\tilde{R}^{(1)}(x, t) \leq \Omega /(\Omega-t)
$$

holds everywhere with equality somewhere at $t=0$. Here we denote by $\tilde{R}^{(1)}(x, t)$ the scalar curvature of the limiting metric $\tilde{g}_{\alpha \bar{\beta}}^{(1)}(x, t)$, and denote $\Omega /(\Omega-t)$ to be 1 if $\Omega=+\infty$. Clearly the limiting solution $\left(\tilde{M}^{(1)}, \tilde{g}_{\alpha \bar{\beta}}^{(1)}(x, t)\right)$ is still noncompact. By Lemma 4.1 and the rescaling invariance of the condition $\left(^{*}\right)$, we see that the limiting solution $\left(\tilde{M}^{(1)}, \tilde{g}_{\alpha \bar{\beta}}^{(1)}(x, t)\right)$ also satisfies

$$
\operatorname{Vol}_{t}\left(\tilde{B}_{t}^{(1)}(x, r)\right) \geq c_{1} r^{2 n}, \quad \text { for all } \quad x \in \tilde{M}^{(1)} \quad \text { and } \quad 0 \leq r<+\infty,
$$

where $\operatorname{Vol}_{t}\left(\tilde{B}_{t}^{(1)}(x, r)\right)$ denotes the volume of the geodesic ball $\tilde{B}_{t}^{(1)}(x, r)$ of radius $r$ centered at $x$ of $\tilde{M}^{(1)}$ with respect to the metric $\tilde{g}_{\alpha \bar{\beta}}^{(1)}(x, t)$.

Denote by $\tilde{d}_{t}^{(1)}\left(x, x_{0}\right)$ the distance between two points $x, x_{0} \in \tilde{M}^{(1)}$ with respect to the metric $\tilde{g}_{\alpha \bar{\beta}}^{(1)}(\cdot, t)$. We first claim that at time $t=0$, we have

$$
\limsup _{\tilde{d}_{0}^{(1)}\left(x, x_{0}\right) \rightarrow+\infty} \tilde{R}^{(1)}(x, 0) \tilde{d}_{0}^{(1)}\left(x, x_{0}\right)=+\infty,
$$


for any fixed $x_{0} \in \tilde{M}^{(1)}$.

Suppose not, thus the curvature of the metric $\tilde{g}_{\alpha \bar{\beta}}^{(1)}(\cdot, 0)$ decays quadratically. By applying a result of Shi (see Theorem 8.2 in [22]), we know that the solution $\tilde{g}_{\alpha \bar{\beta}}^{(1)}(\cdot, t)$ exists for all $t \in(-\infty,+\infty)$ and satisfies

$$
\lim _{t \rightarrow \infty} \sup \left\{\tilde{R}^{(1)}(x, t) \mid x \in \tilde{M}^{(1)}\right\}=0 .
$$

On the other hand, by the Li-Yau-Hamilton inequality of Cao [2], we have

$$
\frac{\partial \tilde{R}^{(1)}}{\partial t} \geq 0, \quad \text { on } \quad \tilde{M}^{(1)} \times(-\infty,+\infty) .
$$

Thus we deduce that $\tilde{R}^{(1)} \equiv 0$ on $\tilde{M}^{(1)} \times(-\infty,+\infty)$ which contradicts with the fact that the scalar curvature $\tilde{R}^{(1)}$ achieves 1 somewhere at $t=0$. This proves the claim (5.2).

With the estimate (5.2) we can then apply a lemma of Hamilton (Lemma 22.2 in [13]) to find a sequence of points $x_{j}, j=1,2, \cdots$, in $\tilde{M}^{(1)}$, a sequence of radii $r_{j}, j=1,2, \cdots$, and a sequence of positive numbers $\delta_{j}, j=1,2, \cdots$, with $\delta_{j} \rightarrow 0$ such that

(a) $\quad \tilde{R}^{(1)}(x, 0) \leq\left(1+\delta_{j}\right) \tilde{R}^{(1)}\left(x_{j}, 0\right)$, for $x \in \tilde{B}_{0}^{(1)}\left(x_{j}, r_{j}\right) ;$

(b) $\quad r_{j}^{2} \tilde{R}^{(1)}\left(x_{j}, 0\right) \rightarrow+\infty$, as $j \rightarrow+\infty$;

(c) if $s_{j}=\tilde{d}_{0}^{(1)}\left(x_{j}, x_{0}\right)$, then $\lambda_{j}=s_{j} / r_{j} \rightarrow+\infty$, as $j \rightarrow+\infty$;

(d) the balls $\tilde{B}_{0}^{(1)}\left(x_{j}, r_{j}\right)$ are disjoint.

Since the metric $\tilde{g}_{\alpha \bar{\beta}}^{(1)}(\cdot, 0)$ has nonnegative curvature operator, the sectional curvature is nonnegative. Denote the minimum of the sectional curvature of the metric $\tilde{g}_{\alpha \bar{\beta}}^{(1)}(\cdot, 0)$ at $x_{j}$ by $\nu_{j}^{(1)}$. We next claim that the following holds

$$
\varepsilon_{j}=\frac{\nu_{j}^{(1)}}{\tilde{R}^{(1)}\left(x_{j}, 0\right)} \rightarrow 0, \text { as } j \rightarrow+\infty .
$$

In fact, suppose not, there exists a subsequence $j_{k} \rightarrow+\infty$ and some positive number $\varepsilon>0$ such that

$$
\varepsilon_{j_{k}}=\frac{\nu_{j_{k}}^{(1)}}{\tilde{R}^{(1)}\left(x_{j_{k}}, 0\right)} \geq \varepsilon, \text { for all } k=1,2, \cdots .
$$


We have seen that the scalar curvature $\tilde{R}^{(1)}(x, t)$ is pointwisely nondecreasing in time. Then by using the local derivative estimate of Shi (see Theorem 13.1 in [13]) and (a), (b), we have

$$
\begin{aligned}
\sup _{x \in \tilde{B}^{(1)}\left(x_{j_{k}}, r_{j_{k}}\right)}\left|\nabla \tilde{R}_{m}^{(1)}(x, 0)\right|^{2} & \leq C\left(\tilde{R}^{(1)}\left(x_{j_{k}}, 0\right)\right)^{2}\left(\frac{1}{r_{j_{k}}^{2}}+\tilde{R}^{(1)}\left(x_{j_{k}}, 0\right)\right) \\
& \leq 2 C\left(\tilde{R}^{(1)}\left(x_{j_{k}}, 0\right)\right)^{3}
\end{aligned}
$$

where $\tilde{R}_{m}^{(1)}$ is the Riemannian curvature tensor of $\tilde{g}_{\alpha \bar{\beta}}^{(1)}$ and $C$ is a positive constant depending only on the dimension.

Denote by $\nu^{(1)}(x)$ the minimum of the sectional curvature of $\tilde{g}_{\alpha \bar{\beta}}^{(1)}(\cdot, 0)$ at $x$. From (5.4), (5.5) and (b) we have

$$
\begin{aligned}
\nu^{(1)}(x) & \geq \nu_{j_{k}}^{(1)}-\sqrt{2 C}\left(\tilde{R}^{(1)}\left(x_{j_{k}}, 0\right)\right)^{\frac{3}{2}} \tilde{d}_{0}^{(1)}\left(x, x_{j_{k}}\right) \\
& \geq \tilde{R}^{(1)}\left(x_{j_{k}}, 0\right)\left(\varepsilon-\sqrt{2 C} \cdot \sqrt{\tilde{R}^{(1)}\left(x_{j_{k}}, 0\right)} \cdot \tilde{d}_{0}^{(1)}\left(x, x_{j_{k}}\right)\right) \\
& \geq \frac{\varepsilon}{2} \tilde{R}^{(1)}\left(x_{j_{k}}, 0\right),
\end{aligned}
$$

as

$$
\tilde{d}_{0}^{(1)}\left(x, x_{j_{k}}\right) \leq \frac{\varepsilon}{2 \sqrt{2 C} \cdot \sqrt{\tilde{R}^{(1)}}\left(x_{j_{k}}, 0\right)}
$$

and $k$ large enough. Thus by combining with (a), there exists $k_{0}>0$ such that for any $k \geq k_{0}$ and $x \in \tilde{B}_{0}^{(1)}\left(x_{j_{k}}, \frac{\varepsilon}{2 \sqrt{2 C} \cdot \sqrt{\tilde{R}^{(1)}\left(x_{j_{k}}, 0\right)}}\right)$, we have

$$
\frac{\varepsilon}{2} \tilde{R}^{(1)}\left(x_{j_{k}}, 0\right) \leq \text { the sectional curvature at } x \leq 2 \tilde{R}^{(1)}\left(x_{j_{k}}, 0\right) .
$$

Therefore the balls $\tilde{B}_{0}^{(1)}\left(x_{j_{k}}, \frac{\varepsilon}{2 \sqrt{2 C} \cdot \sqrt{\tilde{R}\left(x_{j_{k}}, 0\right)}}\right), k_{0} \leq k<+\infty$, are a family of disjoint remote curvature $\beta$-bumps for some $\beta>0$ in the sense of Hamilton [13]. But this contradicts with the finite bumps theorem of Hamilton [13]. So we have proved the claim (5.3).

In the followings we are going to rescale the limiting solution $\left(\tilde{M}^{(1)}, \tilde{g}_{\alpha \bar{\beta}}^{(1)}(x, t)\right)$ along the points $x_{j}, j=1,2, \cdots$. We begin with an injectivity radius estimates. We claim that there exists a positive constant $\alpha$ such that the injectivity radii of $\left(\tilde{M}^{(1)}, \tilde{g}_{\alpha \bar{\beta}}^{(1)}(\cdot, 0)\right)$ at $x_{j}(j=1,2, \cdots)$ are uniformly bounded from below by

$$
\operatorname{inj}_{\tilde{M}^{(1)}}\left(x_{j}, \tilde{g}_{\alpha \bar{\beta}}^{(1)}(\cdot, 0)\right) \geq \frac{\alpha}{\sqrt{\tilde{R}^{(1)}\left(x_{j}, 0\right)}}
$$


for $j=1,2, \cdots$.

We prove the claim by contradiction. Suppose there exists a subsequence $j_{k}$, $k=1,2, \cdots$, such that

$$
\tilde{\varepsilon}_{j_{k}}=\sqrt{\tilde{R}^{(1)}\left(x_{j_{k}}, 0\right)} \cdot i n j_{\tilde{M}^{(1)}}\left(x_{j_{k}}, \tilde{g}_{\alpha \bar{\beta}}^{(1)}(\cdot, 0)\right) \rightarrow 0,
$$

as $k \rightarrow+\infty$. For each $k$, let us choose $x_{j_{k}}$ as the new origin and dilate $\left(\tilde{M}^{(1)}, \tilde{g}_{\alpha \bar{\beta}}^{(1)}(\cdot, 0)\right)$ along $x_{j_{k}}$ such that the dilated manifold, says $\tilde{M}_{k}^{(1)}$, has the injectivity radius 1 at the origin and has the curvature bounded by $2 \tilde{\varepsilon}_{j_{k}}^{2}$ on the ball centered at the origin with the radius not less than $\tilde{r}_{j_{k}}=r_{j_{k}} \sqrt{\tilde{R}^{(1)}\left(x_{j_{k}}, 0\right)}(\rightarrow+\infty$, by (b)). We have seen that the scalar curvature of $\tilde{g}_{\alpha \bar{\beta}}^{(1)}(x, t)$ is pointwisely nondecreasing in time. The curvature bounds on the balls at $t=0$ also give the bounds for previous time in these balls. Then by the local derivative estimate of Shi (see Theorem 13.1 in [13]) and the convergence theorem of Hamilton [12] we know that a subsequence of $\tilde{M}_{k}^{(1)}, k=1,2, \cdots$, converges in $C_{l o c}^{\infty}$ topology to a complete noncompact flat manifold which has the injectivity radius 1 at the origin. But the estimate (5.1) and its rescaling invariance imply that the flat manifold is $\mathbb{R}^{2 n}$ with the Euclidean metric. This contradictions proves the claim (5.6).

Now we let $x_{j}$ be the new origin, dilate the space by a factor $\lambda_{j}$ so that the scalar curvature $\tilde{R}^{(1)}\left(x_{j}, 0\right)$ becomes 1 at the origin at $t=0$, and dilate the time by $\lambda_{j}^{2}$ so that it is still a solution to the Ricci flow. The balls $\tilde{B}_{0}^{(1)}\left(x_{j}, r_{j}\right)$ are dilated to the balls centered at the origins of radii $\tilde{r}_{j}=r_{j} \sqrt{\tilde{R}^{(1)}\left(x_{j}, 0\right)} \rightarrow+\infty$ as $j \rightarrow+\infty$ (by (b)). Since the scalar curvature of the limit $\tilde{g}_{\alpha \bar{\beta}}^{(1)}(x, t)$ is pointwise nondecreasing in time, the curvature bounds on $\tilde{B}_{0}^{(1)}\left(x_{j}, r_{j}\right)$ also give bounds for previous time in these balls. Thus by applying the injectivity radius estimate (5.6) and the compactness theorem of Hamilton [12] again we can get a limit for the dilated solutions. It follows from (5.1), (5.3), (a) and (b) that the limit is a complete noncompact solution, still denoted by $\left(\tilde{M}^{(1)}, \tilde{g}_{\alpha \bar{\beta}}^{(1)}(x, t)\right)$, to the Ricci flow on $t \in(-\infty, 0]$ such that

$(e)_{1}$ the curvature operator is still nonnegative;

$(f)_{1} \quad \tilde{R}^{(1)}(x, t) \leq 1$, for $x \in \tilde{M}^{(1)}, t \in(-\infty, 0]$, and $\tilde{R}^{(1)}(0,0)=1$;

$(g)_{1} \operatorname{Vol}_{t}\left(\tilde{B}_{t}^{(1)}(x, r)\right) \geq c_{1} r^{2 n}$, for all $x \in \tilde{M}^{(1)}, 0 \leq r<+\infty$;

$(h)_{1}$ there exists a 2 -plane at the origin so that at $t=0$, the corresponding sectional curvature vanishes.

If we consider the universal covering of $\tilde{M}^{(1)}$, the induced metric of $\tilde{g}_{\alpha \overline{\bar{\beta}}}^{(1)}(x, t)$ on the universal covering is clearly still a solution to the Ricci flow and satisfies all of 
above $(\mathrm{e})_{1},(\mathrm{f})_{1},(\mathrm{~g})_{1}$, and $(\mathrm{h})_{1}$. Thus, without loss of generality, we may assume that $\tilde{M}^{(1)}$ is simply connected.

Since $\tilde{M}^{(1)}$ is a Kähler manifold, at any point $x$, the curvature operator of the underlying Riemannian manifold restricts to the real $(1,1)$ forms, this is, $\tilde{R}_{m}^{(1)}: \bigwedge_{\mathbb{R}}^{1,1}\left(T_{x} \tilde{M}^{(1)}\right) \rightarrow \bigwedge_{\mathbb{R}}^{1,1}\left(T_{x} \tilde{M}^{(1)}\right)$ and $\tilde{R}_{m}^{(1)} \equiv 0$ on the subspace of $\bigwedge^{2}\left(T_{x} \tilde{M}^{(1)}\right)$ perpendicular to $\bigwedge_{\mathbb{R}}^{1,1}\left(T_{x} \tilde{M}^{(1)}\right)$. This is equivalent to the fact that the Levi-Civita connection of the underlying Riemannian metric on $\tilde{M}^{(1)}$ restricts to the unitary frame bundle. If we define the Lie bracket on $\bigwedge^{2}\left(T_{x} \tilde{M}^{(1)}\right)$ as in [11] by

$$
[\varphi, \psi]_{i j}=g^{k l} \varphi_{i k} \psi_{j l}-g^{k l} \psi_{i k} \varphi_{j l}, \text { where } \varphi, \psi \in \bigwedge^{2}\left(T_{x} \tilde{M}^{(1)}\right),
$$

then $\bigwedge_{\mathbb{R}}^{1,1}\left(T_{x} \tilde{M}^{(1)}\right)$ is a Lie subalgebra of $\bigwedge^{2}\left(T_{x} \tilde{M}^{(1)}\right)$ isomorphic to $u(n)$.

Let us consider the evolution equation of the curvature operator of $\tilde{g}_{\alpha \bar{\beta}}^{(1)}(x, t)$. By applying the strong maximum principle to the evolution equation as in [11] (see Theorem 8.3 of [11]) we know that there exists a positive constant $K$ such that on the time interval $-\infty<t<-K$, the image of the curvature operator of $\left(\tilde{M}^{(1)}, \tilde{g}_{\alpha \bar{\beta}}^{(1)}(\cdot, t)\right)$ at every point $x$ is a fixed Lie subalgebra of $\bigwedge_{\mathbb{R}}^{1,1}\left(T_{x} \tilde{M}^{(1)}\right) \cong u(n)$, invariant under parallel translation and constant in time.

We now want to show that $\tilde{M}^{(1)}$ must be a reducible manifold. Suppose $\tilde{M}^{(1)}$ is an irreducible manifold. Then according to the well-known theorem of Berger [1], $\tilde{M}^{(1)}$ is either a Hermitian symmetric space or has its holonomy group as $U(n), S U(n)$ or $S_{p}\left(\frac{n}{2}\right)$. Since $\tilde{M}^{(1)}$ has nonnegative curvature operator, $\tilde{M}^{(1)}$ is thus either Ricci flat or has $U(n)$ holonomy (see for example [5]). The case of Ricci flat is ruled out by $(\mathrm{e})_{1}$ and $(\mathrm{f})_{1}$. This says that $\tilde{M}^{(1)}$ has $U(n)$ holonomy group. Since $\tilde{M}^{(1)}$ is simply connected, the restricted holonomy group agrees with the full holonomy group. We then have from Ambrose-Singer holonomy theorem that the image of the curvature operator $\tilde{R}_{m}^{(1)}$ at every point $x \in \tilde{M}^{(1)}$ is $u(n) \cong \bigwedge_{\mathbb{R}}^{1,1}\left(T_{x} \tilde{M}^{(1)}\right)$. This, together with the nonnegativity of $\tilde{R}_{m}^{(1)}$, implies that $\tilde{R}_{m}^{(1)}$ is strictly positive when restricted to $\bigwedge_{\mathbb{R}}^{1,1}\left(T_{x} \tilde{M}^{(1)}\right)$.

Let $x$ be an arbitrary point in $\tilde{M}^{(1)}$ and $e_{1}, e_{2}$ be two vectors at $x$. Denote by $J$ the complex structure of $\tilde{M}^{(1)}$. Consider the following (complex) $(1,1)$ vector

$$
\begin{aligned}
& \left(e_{1}+\sqrt{-1} J e_{1}\right) \wedge \overline{\left(e_{2}+\sqrt{-1} J e_{2}\right)} \\
& =\left(e_{1}+\sqrt{-1} J e_{1}\right) \wedge\left(e_{2}-\sqrt{-1} J e_{2}\right) \\
& =\left(e_{1} \wedge e_{2}+J e_{1} \wedge J e_{2}\right)+\sqrt{-1}\left(J e_{1} \wedge e_{2}-e_{1} \wedge J e_{2}\right) .
\end{aligned}
$$


Since $\tilde{R}_{m}^{(1)}$ is strictly positive when restricted to $\bigwedge_{\mathbb{R}}^{1,1}\left(\tilde{M}^{(1)}\right)$, we have

$$
\tilde{R}_{m}^{(1)}\left(e_{1} \wedge e_{2}+J e_{1} \wedge J e_{2}, e_{1} \wedge e_{2}+J e_{1} \wedge J e_{2}\right)>0 .
$$

By the Kählerity of $\tilde{M}^{(1)}$, the sectional curvature $K\left(e_{1}, e_{2}\right)$ of the 2 -plane spanned by $e_{1}, e_{2}$ satisfies

$$
\begin{aligned}
K\left(e_{1}, e_{2}\right) & =\frac{1}{4} \tilde{R}_{m}^{(1)}\left(e_{1} \wedge e_{2}+J e_{1} \wedge J e_{2}, e_{1} \wedge e_{2}+J e_{1} \wedge J e_{2}\right) \\
& >0
\end{aligned}
$$

But the arbitrariness of the point $x$ and the vectors $e_{1}, e_{2}$ gives a contradiction with $(\mathrm{h})_{1}$. So $\tilde{M}^{(1)}$ is a simply connected reducible Kähler manifold.

According to the decomposition theorem of de Rham (see for example Theorem 8.1 in [16]) $\tilde{M}^{(1)}$ can be isometrically splitted as the direct product $\tilde{M}_{1}^{(1)} \times$ $\tilde{M}_{2}^{(2)}$ where each fact is also Kähler. Clearly each factor $\tilde{M}_{i}^{(1)}, i=1,2$, is still a complete noncompact solution of the Ricci flow for $t \in(-\infty, 0]$, has uniformly bounded and nonnegative curvature operator and satisfies the associated Euclidean volume growth, moreover at least one, denoted by $\left(\tilde{M}^{(2)}, \tilde{g}_{\alpha \bar{\beta}}^{(2)}(x, t)\right)$, $t \in(-\infty, 0]$, is not flat. Denote the complex dimension of $\tilde{M}^{(2)}$ by $n_{2}$ which is strictly less than $n$.

Now we repeat the above argument for $\left(\tilde{M}^{(2)}, \tilde{g}_{\alpha \overline{\bar{\beta}}}^{(2)}(x, t)\right), t \in(-\infty, 0]$. For clearity, we outline the main points as followings. We first apply the Li-YauHamilton inequality of Cao [2] and a result of Shi (Theorem 8.2 in [22]) to show that for any fixed $x_{0} \in \tilde{M}^{(2)}$,

$$
\limsup _{\tilde{d}_{0}^{(2)}\left(x, x_{0}\right) \rightarrow+\infty} \tilde{R}^{(2)}(x, 0) \tilde{d}_{0}^{(2)}{ }^{2}\left(x, x_{0}\right)=+\infty .
$$

By using a lemma of Hamilton (Lemma 22.2 in [13]) we can find a sequence of points $x_{j}, j=1,2, \cdots$, in $\tilde{M}^{(2)}$, a sequence of radii $r_{j}, j=1,2, \cdots$, and a sequence of positive number $\delta_{j}, j=1,2, \cdots$, with $\delta_{j} \rightarrow 0$, such that the corresponding (a), (b), (c) and (d) hold. We then apply the finite bumps theorem of Hamilton [13] to show that

$$
\frac{\nu_{j}^{(2)}}{\tilde{R}^{(2)}\left(x_{j}, 0\right)} \rightarrow 0, \text { as } j \rightarrow+\infty,
$$

where $\nu_{j}^{(2)}$ denotes the minimum of the sectional curvature of the metric $\tilde{g}_{\alpha \bar{\beta}}^{(2)}(\cdot, 0)$ at $x_{j}$. Next by combining the property of Euclidean volume growth and a rescaling argument we can show that the injectivity radii of $\left(\tilde{M}^{(2)}, \tilde{g}_{\alpha \bar{\beta}}^{(2)}(\cdot, 0)\right)$ at $x_{j}$ are 
uniformly bounded from below by

$$
\operatorname{inj}_{\tilde{M}^{(2)}}\left(x_{j}, \tilde{g}_{\alpha \bar{\beta}}^{(2)}(\cdot, 0)\right) \geq \frac{\alpha}{\sqrt{\tilde{R}^{(2)}\left(x_{j}, 0\right)}},
$$

for $j=1,2, \cdots$. Here $\alpha$ is some positive constant. Thus we can rescale the solution $\left(\tilde{M}^{(2)}, \tilde{g}_{\alpha \bar{\beta}}^{(2)}(\cdot, t)\right), t \in(-\infty, 0]$, along the points $x_{j}, j=1,2, \cdots$, to get a limit which is a complete noncompact solution, still denoted by $\left(\tilde{M}^{(2)}, \tilde{g}_{\alpha, \bar{\beta}}^{(2)}(x, t)\right)$, to the Ricci flow on $t \in(-\infty, 0]$ such that

$(e)_{2}$ the curvature operator is still nonnegative;

$(f)_{2} \quad \tilde{R}^{(2)}(x, t) \leq 1$, for $x \in \tilde{M}^{(2)}, t \in(-\infty, 0]$, and $\tilde{R}^{(2)}(0,0)=1$;

$(g)_{2}$ there exists some positive constant $c_{2}$ such that

$$
\operatorname{Vol}_{t}\left(\tilde{B}_{t}^{(2)}(x, r)\right) \geq c_{2} r^{2 n_{2}}, \text { for all } x \in \tilde{M}^{(2)}, 0 \leq r<+\infty,
$$

where $\tilde{B}_{t}^{(2)}(x, r)$ is the geodesic ball of $\tilde{M}^{(2)}$ centered at $x$ and with

radius $r$ with respect to the metric $\tilde{g}_{\alpha \bar{\beta}}^{(2)}(\cdot, t)$;

$(h)_{2}$ there exists a 2-plane at the origin so that at $t=0$, the corresponding sectional curvature vanishes.

Without loss of generality, we may assume that $\tilde{M}^{(2)}$ is simply connected. Exactly as before by using the theorem of Berger [1] and the decomposition theorem of de Rham we further deduce that $\tilde{M}^{(2)}$ can be isometrically splitted as the direct product $\tilde{M}_{1}^{(2)} \times \tilde{M}_{2}^{(2)}$. Also each factor $\tilde{M}_{i}^{(2)}, i=1,2$, is still a complete noncompact solution of the Ricci flow for $t \in(-\infty, 0]$, has uniformly bounded and nonnegative curvature operator and satisfies the associated Euclidean volume growth, moreover at least one, denoted by $\left(\tilde{M}^{(3)}, \tilde{g}_{\alpha \bar{\beta}}^{(3)}(x, t)\right), t \in(-\infty, 0]$, is not flat. The complex dimension of $\tilde{M}^{(3)}$ is strictly less than $n_{2}$.

Hence by repeating these procedures we finally obtain a nonflat complex 1 -dimensional complete noncompact solution, denoted by $\left(\tilde{M}, \tilde{g}_{\alpha \bar{\beta}}(x, t)\right)$, of the Ricci flow for $t \in(-\infty, 0]$, which has uniformly bounded and nonnegative curvature and satisfies the following Euclidean volume growth

$$
\operatorname{Vol}_{t}\left(\tilde{B}_{t}(x, r)\right) \geq \tilde{c} r^{2}, \quad \text { for all } x \in \tilde{M}, 0 \leq r<+\infty,
$$

where $\tilde{B}_{t}(x, r)$ denotes the geodesic ball of $\tilde{M}$ centered at $x$ of radius $r$ with respect to the metric $\tilde{g}_{\alpha \bar{\beta}}(\cdot, t)$, and $\tilde{c}$ is some positive constant. As the curvature of $\tilde{g}_{\alpha \bar{\beta}}(x, t)$, is nonnegative, it follows from Cohn-Vossen inequality that

$$
\int_{\tilde{M}} \tilde{R}(x, t) d \sigma_{t} \leq 8 \pi
$$


where $\tilde{R}(x, t)$ is the scalar curvature of $\left(\tilde{M}, \tilde{g}_{\alpha \bar{\beta}}(x, t)\right)$ and $d \sigma_{t}$ is the volume element of the metric $\tilde{g}_{\alpha \bar{\beta}}(x, t)$.

Now the metric $\left.\tilde{g}_{\alpha \bar{\beta}}(x, t)\right)$ is a solution to the Ricci flow on the Riemann surface $\tilde{M}$ over the ancient time interval $(-\infty, 0]$. Thus, (5.8) and (5.9) imply that for each $t<0$, the curvature of $\tilde{g}_{\alpha \bar{\beta}}(x, t)$ has quadratic decay in the average sense of Shi [22], and then the a priori estimate of Shi (see Theorem 8.2 in [22]) implies that the solution $\tilde{g}_{\alpha \bar{\beta}}(x, t)$. exists for all $t \in(-\infty,+\infty)$ and satisfies

$$
\lim _{t \rightarrow+\infty} \sup \{\tilde{R}(x, t) \mid x \in \tilde{M}\}=0 .
$$

Again by the Li-Yau-Hamilton inequality of Cao [2], we conclude that

$$
\tilde{R}(x, t) \equiv 0 \text {, on } \tilde{M} \times(-\infty,+\infty) .
$$

This contradicts with the fact that $\left(\tilde{M}, \tilde{g}_{\alpha \bar{\beta}}(x, t)\right)$ is not flat.

Therefore we have seeked the desired contradiction and have completed the proof of Theorem 5.1.

\section{Curvature Decay in Space}

After obtain the time decay estimate, we can adapt the argument in [8] to obtain the space decay estimates stated in Theorem 3. For sake of completeness, we present a proof as follows.

Proof of Theorem 3. Let $\left(M, g_{\alpha \bar{\beta}}\right)$ be a complex $n$-dimensional complete noncompact Kähler manifold satisfying all the assumptions of Theorem 3. Let $g_{\alpha \bar{\beta}}(x, t)$ be the solution of the Ricci flow (4.1) with $g_{\alpha \bar{\beta}}(x)$ as the initial metric. From Theorem 5.1 we know that the solution $g_{\alpha \bar{\beta}}(x, t)$ exists for all times $t \in$ $[0,+\infty)$ and satisfies

$$
0 \leq R(x, t) \leq \frac{C}{1+t}, \quad \text { on } M \times[0,+\infty),
$$

for some positive constant $C$. Consider the following function

$$
F(x, t)=\log \frac{\operatorname{det}\left(g_{\alpha \bar{\beta}}(x, t)\right)}{\operatorname{det}\left(g_{\alpha \bar{\beta}}(x, 0)\right)}, \quad x \in M, t \geq 0,
$$

introduced in the proof of Lemma 4.1. Recall from the definition of Ricci curvature tensor of a Kähler metric that

$$
\begin{aligned}
R_{\alpha \bar{\beta}}(x, t)-R_{\alpha \bar{\beta}}(x, 0) & =-\partial_{\alpha} \partial_{\bar{\beta}} \log \operatorname{det}\left(g_{\gamma \bar{\delta}}(x, t)\right)+\partial_{\alpha} \partial_{\bar{\beta}} \log \operatorname{det}\left(g_{\gamma \bar{\delta}}(x, 0)\right) \\
& =-\partial_{\alpha} \partial_{\bar{\beta}} F(x, t) .
\end{aligned}
$$


Thus

$$
R(x, 0)=\Delta_{0} F(x, t)+g^{\alpha \bar{\beta}}(x, 0) R_{\alpha \bar{\beta}}(x, t), \quad x \in M, t \geq 0,
$$

where $\Delta_{0}$ is the Laplacian operator of the metric $g_{\alpha \bar{\beta}}(x, 0)$.

Since $\left(M, g_{\alpha \bar{\beta}}(\cdot, 0)\right)$ has nonnegative Ricci curvature and is of Euclidean volume growth, it is well known (see for example [20]) that the Green function $G_{0}(x, y)$ of the initial metric $g_{\alpha \bar{\beta}}(\cdot, 0)$ exists on $M$ and satisfies the estimates

$$
\frac{C_{4}^{-1}}{d_{0}^{2 n-2}(x, y)} \leq G_{0}(x, y) \leq \frac{C_{4}}{d_{0}^{2 n-2}(x, y)}
$$

and

$$
\left|\nabla_{y} G_{0}(x, y)\right|_{0} \leq \frac{C_{4}}{d_{0}^{2 n-1}(x, y)},
$$

where $d_{0}(x, y),|\cdot|_{0}$ are the geodesic distance of $x, y$ and the norm with respect to the initial metric $g_{\alpha \bar{\beta}}(\cdot, 0)$, and $C_{4}$ is some positive constant.

For any fixed $\bar{x}_{0} \in M$ and any $\alpha>0$, we denote

$$
\Omega_{\alpha}=\left\{x \in M \mid G_{0}\left(\bar{x}_{0}, x\right) \geq \alpha\right\} .
$$

By (6.3), it is not hard to see

$$
B_{0}\left(\bar{x}_{0},\left(\frac{C_{4}^{-1}}{\alpha}\right)^{\frac{1}{2 n-2}}\right) \subset \Omega_{\alpha} \subset B_{0}\left(\bar{x}_{0},\left(\frac{C_{4}}{\alpha}\right)^{\frac{1}{2 n-2}}\right) .
$$

Recall from (4.4) that

$$
\frac{\partial F(x, t)}{\partial t}=-R(x, t), \quad x \in M, t \geq 0 .
$$

By combining with the time decay estimate (6.1) we deduce that

$$
0 \geq F(x, t) \geq-C_{5} \log (1+t), \quad x \in M, t \geq 0,
$$

for some positive constant $C_{5}$.

Multiplying (6.2) by $G_{0}\left(\bar{x}_{0}, x\right)-\alpha$ and integrating over $\Omega_{\alpha}$, we have

$$
\int_{\Omega_{\alpha}} R(x, 0)\left(G_{0}\left(\bar{x}_{0}, x\right)-\alpha\right) d x
$$




$$
\begin{aligned}
& =\int_{\Omega_{\alpha}}\left(\Delta_{0} F(x, t)\right)\left(G_{0}\left(\bar{x}_{0}, x\right)-\alpha\right) d x \\
& \quad+\int_{\Omega_{\alpha}} g^{\alpha \bar{\beta}}(x, 0) R_{\alpha \bar{\beta}}(x, t)\left(G\left(\bar{x}_{0}, x\right)-\alpha\right) d x \\
& =-\int_{\partial \Omega_{\alpha}} F(x, t) \frac{\partial G_{0}\left(\bar{x}_{0}, x\right)}{\partial \nu} d \sigma-F\left(\bar{x}_{0}, t\right) \\
& \quad+\int_{\Omega_{\alpha}} g^{\alpha \bar{\beta}}(x, 0) R_{\alpha \bar{\beta}}(x, t)\left(G\left(\bar{x}_{0}, x\right)-\alpha\right) d x \\
& \leq C_{5}\left(1+C_{4}^{1+\frac{2 n-1}{2 n-2}} \alpha^{\frac{2 n-1}{2 n-2}} V o l_{0}\left(\partial \Omega_{\alpha}\right)\right) \log (1+t) \\
& \quad+\int_{\Omega_{\alpha}} g^{\alpha \bar{\beta}}(x, 0) R_{\alpha \bar{\beta}}(x, t) G_{0}\left(\bar{x}_{0}, x\right) d x,
\end{aligned}
$$

by (6.3), (6.4) and (6.6). Here we used $\nu$ to denote the outer unit normal of $\partial \Omega_{\alpha}$. From the coarea formula, (6.3), (6.4) and (6.5), we have

$$
\begin{aligned}
\frac{1}{\alpha} \int_{\alpha}^{2 \alpha} r^{\frac{2 n-1}{2 n-2}} \operatorname{Vol}_{0}\left(\partial \Omega_{r}\right) d r & \leq 2^{\frac{2 n-1}{2 n-2}} \alpha^{\frac{1}{2 n-2}} \int_{\alpha}^{2 \alpha} \int_{\partial \Omega_{r}}\left|\nabla G_{0}\left(\bar{x}_{0}, x\right)\right|_{0} d \sigma|d \nu| \\
& \leq 2^{\frac{2 n-1}{2 n-2}} \cdot C_{4}^{1+\frac{2 n-1}{2 n-2}} \cdot \alpha^{\frac{2 n}{2 n-2}} \operatorname{Vol}_{0}\left(\Omega_{\alpha}\right) \\
& \leq 2^{\frac{2 n-1}{2 n-2}} \cdot C_{4}^{1+\frac{2 n-1}{2 n-2}} \alpha^{\frac{2 n}{2 n-2}} \operatorname{Vol}_{0}\left(B_{0}\left(\bar{x}_{0},\left(\frac{C_{4}}{\alpha}\right)^{\frac{1}{2 n-2}}\right)\right. \\
& \leq C_{6}
\end{aligned}
$$

for some positive constant $C_{6}$ by the standard volume comparison theorem. Integrating (6.7) from $\alpha$ to $2 \alpha$ and using the above inequality, we get

$$
\begin{aligned}
\int_{\Omega_{\alpha}} R(x, 0)\left(G_{0}\left(\bar{x}_{0}, x\right)-2 \alpha\right) d x \leq & C_{5}\left(1+C_{4}^{1+\frac{2 n-1}{2 n-2}} \cdot C_{6}\right) \log (1+t) \\
& +\int_{\Omega_{\alpha}} g^{\alpha \bar{\beta}}(x, 0) R_{\alpha \bar{\beta}}(x, t) G_{0}\left(\bar{x}_{0}, x\right) d x .
\end{aligned}
$$

It is easy to see that

$$
\int_{\Omega_{4 \alpha}} R(x, 0) G_{0}\left(\bar{x}_{0}, x\right) d x \leq 2 \int_{\Omega_{2 \alpha}} R(x, 0)\left(G_{0}\left(\bar{x}_{0}, x\right)-2 \alpha\right) d x,
$$

and by the equation of the Ricci flow (4.1), we have

$$
\int_{0}^{t} \int_{\Omega_{\alpha}} g^{\alpha \bar{\beta}}(x, 0) R_{\alpha \bar{\beta}}(x, t) G_{0}\left(\bar{x}_{0}, x\right) d x d t
$$




$$
\begin{aligned}
& =\int_{\Omega_{\alpha}} g^{\alpha \bar{\beta}}(x, 0)\left(g_{\alpha \bar{\beta}}(x, 0)-g_{\alpha \bar{\beta}}(x, t)\right) G_{0}\left(\bar{x}_{0}, x\right) d x \\
& \leq n \int_{\Omega_{\alpha}} G_{0}\left(\bar{x}_{0}, x\right) d x .
\end{aligned}
$$

Thus by integrating (6.8) in time from 0 to $t$ and combining the above two inequalites, we get for any $t>0$,

$$
\int_{\Omega_{4 \alpha}} R(x, 0) G_{0}\left(\bar{x}_{0}, x\right) d x \leq 2 C_{5}\left(1+C_{4}^{1+\frac{2 n-1}{2 n-2}} \cdot C_{6}\right) \log (1+t)+\frac{2 n}{t} \int_{\Omega_{\alpha}} G_{0}\left(\bar{x}_{0}, x\right) d x .
$$

Finally, substituting (6.3) and (6.5) into the above inequality, we see that there exists some positive constant $C_{7}$ such that for any $\bar{x}_{0} \in M, t>0$ and $r>0$,

$$
\int_{B_{0}\left(\bar{x}_{0}, r\right)} \frac{R(x, 0)}{d_{0}^{2 n-2}\left(\bar{x}_{0}, x\right)} d x \leq C_{7}\left(\log (1+t)+\frac{r^{2}}{t}\right) .
$$

By choosing $t=r^{2}$ we get the desired first estimate. The second estimate is a direct consequence of first estimate.

\section{Examples of Positively Curved Kähler}

\section{Manifolds}

A homothetically expanding gradient Kähler-Ricci soliton in a manifold $M$ is a complete solution of the Ricci flow which moves along the equation (4.1) by a oneparameter group of biholomorphisms in the direction of a gradient holomorphic vector field and also expands by a factor at the same time. More precisely, this means that the Ricci tensor of $g_{\alpha \bar{\beta}}$ can be expressed as

$$
\left\{\begin{aligned}
R_{\alpha \bar{\beta}} & =\nabla_{\alpha} \nabla_{\bar{\beta}} f-\rho g_{\alpha \bar{\beta}}, \\
\nabla_{\alpha} \nabla_{\beta} f & =\nabla_{\bar{\alpha}} \nabla_{\bar{\beta}} f=0
\end{aligned}\right.
$$

for some constant $\rho>0$ and some function $f$ on $M$. In [4], Cao obtained a family of homothetically expanding gradient Kähler-Ricci soliton on $\mathbb{C}^{n}$ of positive sectional curvature by constructing global potential functions on $\mathbb{C}^{n}$. In this section we will further show that this family of Kähler-Ricci solitons actually have nonnegative curvature operator everywhere and have positive curvature operator when restricted on the subspace of $(1,1)$-forms. Moreover the soliton metrics have Euclidean volume growth and their curvatures are quadratic decay. 
To begin we recall the construction of the Kähler-Ricci solitons on $\mathbb{C}^{n}$ in [4]. Let $z_{1}, z_{2}, \cdots, z_{n}$ denote the coordinate functions on $\mathbb{C}^{n}$ and denote $|z|=$ $\sqrt{\left|z_{1}\right|^{2}+\cdots+\left|z_{n}\right|^{2}}$ the distance function from the origin $O$ with the Euclidean metric on $\mathbb{C}^{n}$. Let $t=\log |z|^{2}$ and also let $u: \mathbb{R} \rightarrow \mathbb{R}$ be a smooth, convex and increasing function. The Kähler potential function $u(t)$ on $\mathbb{C}^{n}$ induces a Kähler metric

$$
\begin{aligned}
g_{\alpha \bar{\beta}} & =\partial_{\alpha} \partial_{\bar{\beta}} u(t) \\
& =e^{-t} u^{\prime} \delta_{\alpha \beta}+e^{-2 t} z_{\beta} \bar{z}_{\alpha}\left(u^{\prime \prime}-u^{\prime}\right),
\end{aligned}
$$

on $\mathbb{C}^{n}$. It then follows easily that

$$
g^{\alpha \bar{\beta}}=e^{t}\left(u^{\prime}\right)^{-1} \delta_{\alpha \beta}+\bar{z}_{\beta} z_{\alpha}\left(\left(u^{\prime \prime}\right)^{-1}-\left(u^{\prime}\right)^{-1}\right)
$$

and

$$
\operatorname{det}\left(g_{\alpha \bar{\beta}}\right)=e^{-n t} u^{\prime \prime}\left(u^{\prime}\right)^{n-1}
$$

Set

$$
\begin{aligned}
f(t) & =-\log \operatorname{det}\left(g_{\alpha \bar{\beta}}\right) \\
& =n t-(n-1) \log u^{\prime}-\log u^{\prime \prime} .
\end{aligned}
$$

From the definition of Ricci curvature,

$$
R_{\alpha \bar{\beta}}+g_{\alpha \bar{\beta}}=\partial_{\alpha} \partial_{\bar{\beta}}(f+u) .
$$

Consider the gradient vector field given by

$$
\begin{aligned}
V^{\alpha} & =g^{\alpha \bar{\beta}} \partial_{\bar{\beta}}(f+u) \\
& =\left(e^{t}\left(u^{\prime}\right)^{-1} z_{\alpha}+e^{t} z_{\alpha}\left(\left(u^{\prime \prime}\right)^{-1}-\left(u^{\prime}\right)^{-1}\right)\right)\left(f^{\prime}+u^{\prime}\right) e^{-t} \\
& =\frac{f^{\prime}+u^{\prime}}{u^{\prime \prime}} z_{\alpha},
\end{aligned}
$$

which is holomorphic if and only if $\frac{f^{\prime}+u^{\prime}}{u^{\prime \prime}}$ is constant.

Thus the Kähler metric $g_{\alpha \bar{\beta}}=\partial_{\alpha} \partial_{\bar{\beta}} u(t)$ is a solution of (7.1) with $\rho=1$ if and only if

$$
f^{\prime}+u^{\prime}=\lambda u^{\prime \prime}
$$

for some constant $\lambda$. Now by letting $u^{\prime}=\phi$, the equation (7.1) with $\rho=1$ for expanding Kähler-Ricci solitons is reduced to

$$
\frac{\phi^{\prime \prime}}{\phi^{\prime}}+\left(\frac{n-1}{\phi}+\lambda\right) \phi^{\prime}=n+\phi
$$


i.e.,

$$
\left(\log \left(\phi^{\prime} \phi^{n-1} e^{\lambda \phi}\right)\right)^{\prime}=n+\phi
$$

and then

$$
\phi^{\prime}=\frac{1}{\lambda^{n+1} \phi^{n-1}}\left(\lambda^{n} \phi^{n}+\sum_{j=0}^{n-1}(-1)^{n-j} \frac{n !}{j !}(1-\lambda)(\lambda \phi)^{j}+c e^{-\lambda \phi}\right)
$$

where $c$ is a constant. It was shown in [4] that for each $\lambda>1$ and by choosing $c=(-1)^{n-1} n !(1-\lambda)$, the ODE (7.8) has a solution with $\phi>0$ and $\phi^{\prime}>0$ such that the Kähler metric $g_{\alpha \bar{\beta}}=\partial_{\alpha} \partial_{\bar{\beta}} u(t)$ is complete on $\mathbb{C}^{n}$ and has positive sectional curvature. In order to show these Kähler metrics actually having positive curvature operator on the subspace of $(1,1)$-forms, we compute the curvature tensors as follows.

By (7.2) and (7.3), a straightforward computation gives

$$
\begin{aligned}
\frac{\partial g_{\alpha \bar{\beta}}}{\partial z_{\gamma}}= & e^{-2 t}\left(u^{\prime \prime}-u^{\prime}\right)\left(\bar{z}_{\alpha} \delta_{\beta \gamma}+\bar{z}_{\gamma} \delta_{\alpha \beta}\right)+e^{-3 t}\left(u^{(3)}-3 u^{\prime \prime}+2 u^{\prime}\right) \bar{z}_{\gamma} z_{\beta} \bar{z}_{\alpha} \\
\frac{\partial^{2} g_{\alpha \bar{\beta}}}{\partial z_{\gamma} \partial \bar{z}_{\eta}}= & e^{-2 t}\left(u^{\prime \prime}-u^{\prime}\right)\left(\delta_{\alpha \eta} \delta_{\beta \gamma}+\delta_{\alpha \beta} \delta_{\gamma \eta}\right)+e^{-3 t}\left(u^{(3)}-3 u^{\prime \prime}+2 u^{\prime}\right) \times \\
& {\left[z_{\eta} \bar{z}_{\alpha} \delta_{\beta \gamma}+z_{\eta} \bar{z}_{\gamma} \delta_{\alpha \beta}+z_{\beta} \bar{z}_{\alpha} \delta_{\gamma \eta}+\bar{z}_{\gamma} z_{\beta} \delta_{\alpha \eta}\right] } \\
& +e^{-4 t}\left(u^{(4)}-6 u^{(3)}+11 u^{\prime \prime}-6 u^{\prime}\right) z_{\eta} \bar{z}_{\gamma} z_{\beta} \bar{z}_{\alpha}
\end{aligned}
$$

and

$$
\begin{aligned}
R_{\alpha \bar{\beta} \gamma \bar{\eta}}= & -\frac{\partial^{2} g_{\alpha \bar{\beta}}}{\partial z_{\gamma} \partial \bar{z}_{\eta}}+g^{\xi \bar{\zeta}} \frac{\partial g_{\xi \bar{\beta}}}{\partial \bar{z}_{\eta}} \frac{\partial g_{\alpha \bar{\zeta}}}{\partial z_{\gamma}} \\
= & -e^{2 t}\left(u^{\prime \prime}-u^{\prime}\right)\left(\delta_{\alpha \eta} \delta_{\beta \gamma}+\delta_{\alpha \beta} \delta_{\gamma \eta}\right) \\
& +e^{-3 t}\left(-\left(u^{(3)}-3 u^{\prime \prime}+2 u^{\prime}\right)+\left(u^{\prime}\right)^{-1}\left(u^{\prime \prime}-u^{\prime}\right)^{2}\right) \times \\
& \left(\bar{z}_{\alpha} z_{\eta} \delta_{\beta \gamma}+z_{\eta} \bar{z}_{\gamma} \delta_{\alpha \beta}+z_{\beta} \bar{z}_{\alpha} \delta_{\gamma \eta}+\bar{z}_{\gamma} z_{\beta} \delta_{\alpha \eta}\right) \\
& +e^{-4 t}\left[\left(u^{\prime}\right)^{-1}\left(u^{(3)}-3 u^{\prime \prime}+2 u^{\prime}\right)\left(u^{(3)}+u^{\prime \prime}-2 u^{\prime}\right)+\left(\left(u^{\prime \prime}\right)^{-1}\right.\right. \\
& \left.\left.-\left(u^{\prime}\right)^{-1}\right)\left(u^{(3)}-u^{\prime \prime}\right)^{2}-\left(u^{(4)}-6 u^{(3)}+11 u^{\prime \prime}-6 u^{\prime}\right)\right] \cdot \bar{z}_{\alpha} z_{\beta} \bar{z}_{\gamma} z_{\eta}
\end{aligned}
$$

Note that the Kähler metric $g_{\alpha \bar{\beta}}=\partial_{\alpha} \partial_{\bar{\beta}} u(t)$ is rotationally symmetric on $\mathbb{C}^{n}$. To facilitate computations, we only need to compute the curvature at a point 
$z=(r, 0, \cdots, 0)$. Let $\xi^{\alpha \bar{\beta}}$ be any nonzero $(1,1)$-vector at the point. From a straightforward computation we get

$$
\begin{aligned}
R_{\alpha \bar{\beta} \gamma \bar{\eta}} \xi^{\alpha \bar{\beta} \overline{\xi^{\gamma \bar{\eta}}}}= & e^{-2 t}\left[a\left(\left|\sum_{\alpha=1}^{n} \xi^{\alpha \bar{\alpha}}\right|^{2}+\sum_{\alpha, \beta=1}^{n}\left|\xi^{\alpha \bar{\beta}}\right|^{2}\right)\right. \\
& +b\left(\sum_{\alpha=1}^{n}\left|\xi^{1 \bar{\alpha}}\right|^{2}+\overline{\xi^{1 \overline{1}}}\left(\sum_{\alpha=1}^{n} \xi^{\alpha \bar{\alpha}}\right)+\xi^{1 \overline{1}}\left(\overline{\left.\sum_{\alpha=1}^{n} \xi^{\alpha \bar{\alpha}}\right)}\right.\right. \\
& \left.\left.+\sum_{\alpha=1}^{n}\left|\xi^{\alpha \overline{1}}\right|^{2}\right)+c\left|\xi^{1 \overline{1}}\right|^{2}\right] \\
= & e^{-2 t}\left[(c+2 a+4 b)\left|\xi^{1 \overline{1}}\right|^{2}+(a+b)\left(\xi ^ { 1 \overline { 1 } } \left(\overline{\left.\sum_{\alpha=2}^{n} \xi^{\alpha \bar{\alpha}}\right)}\right.\right.\right. \\
& +\left(\sum_{\alpha=2}^{n} \xi^{\alpha \bar{\alpha}}\right) \overline{\xi^{1 \overline{1}}}+(a+b)\left(\sum_{\alpha=2}^{n}\left|\xi^{1 \bar{\alpha}}\right|^{2}+\sum_{\alpha=2}^{n}\left|\xi^{\alpha \overline{1}}\right|^{2}\right) \\
& +a\left(\left|\sum_{\alpha=2}^{n} \xi^{\alpha \bar{\alpha}}\right|^{2}+\sum_{\alpha, \beta=2}^{n}\left|\xi^{\alpha \bar{\beta}}\right|^{2}\right),
\end{aligned}
$$

where the functions $a, b$ and $c$ are defined by

$$
\begin{aligned}
a= & \phi-\phi^{\prime}, \\
b= & \phi^{-1}\left(\phi^{\prime}-\phi\right)^{2}-\left(\phi^{\prime \prime}-3 \phi^{\prime}+2 \phi\right), \\
c= & \phi^{-1}\left(\phi^{\prime \prime}-3 \phi^{\prime}+2 \phi\right)\left(\phi^{\prime \prime}+\phi^{\prime}-2 \phi\right)+\left(\left(\phi^{\prime}\right)^{-1}-\phi^{-1}\right)\left(\phi^{\prime \prime}-\phi^{\prime}\right)^{2} \\
& -\left(\phi^{(3)}-6 \phi^{\prime \prime}+11 \phi^{\prime}-6 \phi\right) .
\end{aligned}
$$

By Cauchy-Schwarz inequality, we see

$$
\begin{aligned}
R_{\alpha \bar{\beta} \gamma \bar{\eta}} \xi^{\alpha \bar{\beta}} \overline{\xi^{\gamma \bar{\eta}}} \geq & e^{-2 t}\left[(c+2 a+4 b)\left|\xi^{1 \overline{1}}\right|^{2}+(a+b)\left(\xi^{1 \overline{1}}\left(\sum_{\alpha=2}^{n} \xi^{\alpha \bar{\alpha}}\right)\right.\right. \\
& +\left(\sum_{\alpha=2}^{n} \xi^{\alpha \bar{\alpha}}\right) \overline{\xi^{1 \overline{1}}}+(a+b)\left(\sum_{\alpha=2}^{n}\left|\xi^{1 \bar{\alpha}}\right|^{2}+\sum_{\alpha=2}^{n}\left|\xi^{\alpha \overline{1}}\right|^{2}\right) \\
& \left.+\frac{n}{n-1} a\left|\sum_{\alpha=2}^{n} \xi^{\alpha \bar{\alpha}}\right|^{2}\right],
\end{aligned}
$$

and we also see

$$
a+b=\phi^{-1}\left(\left(\phi^{\prime}\right)^{2}-\phi \phi^{\prime \prime}\right),
$$




$$
c+2 a+4 b=\left(\phi^{\prime}\right)^{-1}\left(\left(\phi^{\prime \prime}\right)^{2}-\phi^{\prime} \phi^{(3)}\right) .
$$

Thus to show that the curvature operator is strictly positive on the subspace $\bigwedge^{1,1}$ of $(1,1)$-forms we only need to verify the following inequalities:
(A) $\quad a>0$
(B) $\quad a+b>0$
(C) $\quad c+2 a+4 b>0$;
(D) $\quad(a+b)^{2}<\frac{n}{n-1} a \cdot(c+2 a+4 b)$.

VerificAtion of (A). Recall from (7.8) with $c=(-1)^{n+1} n !(1-\lambda)$ that

$$
\begin{aligned}
\phi^{\prime} & =\frac{1}{\lambda^{n+1} \phi^{n-1}}\left(\lambda^{n} \phi^{n}+(-1)^{n+1} n !(1-\lambda) \sum_{j=n}^{\infty} \frac{(-\lambda \phi)^{j}}{j !}\right) \\
& =\frac{1}{\lambda^{n+1} \phi^{n-1}}\left(\lambda^{n+1} \phi^{n}+(-1)^{n+1} n !(1-\lambda) \sum_{j=n+1}^{\infty} \frac{(-\lambda \phi)^{j}}{j !}\right) .
\end{aligned}
$$

We thus have

$$
\begin{aligned}
a & =\phi-\phi^{\prime} \\
& =\frac{(\lambda-1) n !}{\lambda^{n+1} \phi^{n-1}} \cdot(-1)^{n+1} \sum_{j=n+1}^{\infty} \frac{(-\lambda \phi)^{j}}{j !} .
\end{aligned}
$$

Denote by

$$
f_{k}(x)=(-1)^{k} \sum_{j=k}^{\infty} \frac{(-x)^{j}}{j !}, \quad k=0,1, \cdots, n+1, \quad \text { for } x \geq 0 .
$$

Clearly

$$
f_{n+1}^{\prime}=f_{n}, \quad f_{n}^{\prime}=f_{n-1}, \quad \cdots, \quad f_{2}^{\prime}=f_{1}, \quad f_{1}^{\prime}=f_{0}=e^{-x}>0 .
$$

Then we deduce from (7.11) that

$$
a=\frac{(\lambda-1) n !}{\lambda^{n+1} \phi^{n-1}} f_{n+1}(\lambda \phi)>0, \quad \text { as } \lambda>1 .
$$

Verification of (B). By (7.7) and (7.11) we have

$$
\begin{aligned}
\phi^{\prime \prime} \phi-\left(\phi^{\prime}\right)^{2} & =(n+\phi) \phi \phi^{\prime}-(n+\lambda \phi)\left(\phi^{\prime}\right)^{2} \\
& =\frac{(\lambda-1) \phi^{\prime}}{\lambda^{n+1} \phi^{n-1}}\left[(n+\lambda \phi) n ! f_{n+1}(\lambda \phi)-(\lambda \phi)^{n+1}\right] .
\end{aligned}
$$


Denote by

$$
g_{k}(x)=(k-1+x)(k-1) ! f_{k}(x)-x^{k}, \quad k=1, \cdots, n+1, \quad \text { for } x \geq 0 .
$$

It is easy to check that

$$
g_{n+1}^{\prime}=n g_{n}, \quad g_{n}^{\prime}=(n-1) g_{n-2}, \quad \cdots, \quad g_{2}^{\prime}=g_{1}=-x e^{-x} \leq 0 .
$$

Then we deduce that

$$
\begin{aligned}
a+b & =\phi^{-1}\left(\left(\phi^{\prime}\right)^{2}-\phi \phi^{\prime \prime}\right) \\
& =\frac{(\lambda-1) \phi^{\prime}}{\lambda^{n+1} \phi^{n-1}}\left(-g_{n+1}(\lambda \phi)\right) \\
& >0, \quad \text { as } \lambda>1 .
\end{aligned}
$$

VERIFICATION OF (C). By (7.7) and (7.10) we have

$$
\begin{aligned}
\frac{\phi^{(3)} \phi^{\prime}-\left(\phi^{\prime \prime}\right)^{2}}{\left(\phi^{\prime}\right)^{2}=} & \left(\frac{\phi^{\prime \prime}}{\phi^{\prime}}\right)^{\prime} \\
= & \left(n+\phi-\left(\frac{n-1}{\phi}+\lambda\right) \phi^{\prime}\right)^{\prime} \\
= & \frac{\phi^{\prime}}{\phi^{2}}\left[\phi^{2}+\left(n-1+(n-1+\lambda \phi)^{2}\right) \phi^{\prime}\right. \\
& -(n-1+\lambda \phi)(n+\phi) \phi] \\
= & \frac{\phi^{\prime}}{\phi^{2}}\left[\phi^{2}+\left(n-1+(n-1+\lambda \phi)^{2}\right) \frac{1}{\lambda^{n+1} \phi^{n-1}}\left(\lambda^{n+1} \phi^{n}\right.\right. \\
& \left.\left.-(\lambda-1) n ! f_{n+1}(\lambda \phi)\right)-(n-1+\lambda \phi)(n+\phi) \phi\right] \\
= & \frac{\phi^{\prime}(\lambda-1)}{(\lambda \phi)^{n+1}}\left[(\lambda \phi)^{n+1}(n-2+\lambda \phi)\right. \\
& \left.-n !\left(n-1+(n-1+\lambda \phi)^{2}\right) f_{n+1}(\lambda \phi)\right] .
\end{aligned}
$$

Note that

$$
f_{n}(x)=\frac{x^{n}}{n !}-f_{n+1}(x), \quad \text { for } x \geq 0 .
$$

We thus have

$$
\begin{aligned}
\frac{\phi^{(3)} \phi^{\prime}-\left(\phi^{\prime \prime}\right)^{2}}{\left(\phi^{\prime}\right)^{2}}= & \frac{(\lambda-1) \phi^{\prime}}{(\lambda \phi)^{n+1}}\left[\left(n-1+(n-1+\lambda \phi)^{2}\right) n ! f_{n}(\lambda \phi)\right. \\
& \left.-n(n-1+\lambda \phi)(\lambda \phi)^{n}\right] .
\end{aligned}
$$


Denote by

$$
h_{k}(x)=\left(k-1+(k-1+x)^{2}\right) k ! f_{k}(x)-k(k-1+x) x^{n},
$$

$$
k=1,2, \cdots, n, \quad \text { for } x \geq 0 .
$$

It is easy to check that

$$
h_{n}^{\prime}=n h_{n-1}, \quad h_{n-1}^{\prime}=(n-1) h_{n-2}, \quad \cdots, \quad h_{2}^{\prime}=2 h_{1}=2 x^{2}\left(-e^{-x}\right) \leq 0 .
$$

Thus we deduce from (7.14) that

$$
\begin{aligned}
c+2 a+4 b & =\left(\phi^{\prime}\right)^{-1}\left(\left(\phi^{\prime \prime}\right)^{2}-\phi^{\prime} \phi^{(3)}\right) \\
& =\frac{(\lambda-1)\left(\phi^{\prime}\right)^{2}}{(\lambda \phi)^{n+1}}\left(-h_{n}(\lambda \phi)\right) \\
& >0, \quad \text { as } \lambda>1 .
\end{aligned}
$$

VERIFICATION OF (D). From (7.12), (7.13) and (7.15), we have

$$
\frac{n}{n-1} a \cdot(c+2 a+4 b)-(a+b)^{2}
$$




$$
\begin{aligned}
& =\frac{\left(\phi^{\prime}\right)^{2}(\lambda-1)^{2}}{\left(\lambda^{n+1} \phi^{n}\right)^{2}}\left[\frac { n } { n - 1 } n ! f _ { n + 1 } ( \lambda \phi ) \left(n(n-1+\lambda \phi)(\lambda \phi)^{n}\right.\right. \\
& \left.-\left(n-1+(n-1+\lambda \phi)^{2}\right) n ! f_{n}(\lambda \phi)\right) \\
& \left.-\left((\lambda \phi)^{n+1}-(n+\lambda \phi) n ! f_{n+1}(\lambda \phi)\right)^{2}\right] \\
& =\frac{\left(\phi^{\prime}\right)^{2}(\lambda-1)^{2}}{\left(\lambda^{n+1} \phi^{n}\right)^{2}}\left[\frac { n } { n - 1 } n ! ( \frac { ( \lambda \phi ) ^ { n } } { n ! } - f _ { n } ( \lambda \phi ) ) \left(n(n-1+\lambda \phi)(\lambda \phi)^{n}\right.\right. \\
& \left.-\left(n-1+(n-1+\lambda \phi)^{2}\right) n ! f_{n}(\lambda \phi)\right) \\
& -\left((\lambda \phi)^{n+1}-(n+\lambda \phi) n !\left(\frac{(\lambda \phi)^{n}}{n !}-f_{n}(\lambda \phi)\right)^{2}\right] \\
& =\frac{\left(\phi^{\prime}\right)^{2}(\lambda-1)^{2}}{\left(\lambda^{n+1} \phi^{n}\right)^{2}}\left[\frac { n } { n - 1 } \left(n(n-1+\lambda \phi)(\lambda \phi)^{2 n}\right.\right. \\
& -\left(\left(n-1+(n-1+\lambda \phi)^{2}\right)+n(n-1+\lambda \phi)\right)(\lambda \phi)^{n} n ! f_{n}(\lambda \phi) \\
& \left.+(n !)^{2}\left(f_{n}(\lambda \phi)\right)^{2}\left(n-1+(n-1+\lambda \phi)^{2}\right)\right)-(n+\lambda \phi)^{2}(n !)^{2}\left(f_{n}(\lambda \phi)\right)^{2} \\
& \left.-2(n+\lambda \phi) n ! f_{n}(\lambda \phi) \cdot n(\lambda \phi)^{n}+n^{2}(\lambda \phi)^{2 n}\right] \\
& =\frac{n^{2}\left(\phi^{\prime}\right)^{2}(\lambda-1)^{2}}{(n-1)\left(\lambda^{n+1} \phi^{n}\right)^{2}}\left[\left((n-1) ! f_{n}(\lambda \phi)\right)^{2}(n(n-1\right. \\
& \left.\left.+(n-1+\lambda \phi)^{2}\right)-(n-1)(n+\lambda \phi)^{2}\right) \\
& -(n-1) ! f_{n}(\lambda \phi)\left(\left(n-1+(n-1+\lambda \phi)^{2}\right.\right. \\
& \left.\left.+n(n-1+\lambda \phi))(\lambda \phi)^{n}-2(n-1)(n+\lambda \phi)(\lambda \phi)^{n}\right)+(\lambda \phi)^{2 n+1}\right] \\
& =\frac{n^{2}\left(\phi^{\prime}\right)^{2}(\lambda-1)^{2}\left(f_{n}(\lambda \phi)\right)^{2}}{(n-1)\left(\lambda^{n+1} \phi^{n}\right)^{2}}\left[\left((n-1) ! f_{n}(\lambda \phi)\right)^{2}\right. \\
& \left.-(n-1) ! f_{n}(\lambda \phi)\left((\lambda \phi)^{n}+n(\lambda \phi)^{n-1}\right)+(\lambda \phi)^{2 n-1}\right] .
\end{aligned}
$$

Thus to verify (D) it is sufficient to prove the following inequality

$$
\left((n-1) ! f_{n}(x)\right)^{2}-(n-1) ! f_{n}(x)\left(x^{n}+n x^{n-1}\right)+x^{2 n-1}>0,
$$

as $x>0$.

Set

$$
L(x)=\left((n-1) ! e^{x} f_{n}(x)\right)^{2}-e^{x}\left(x^{n}+n x^{n-1}\right)\left((n-1) ! e^{x} f_{n}(x)\right)+e^{2 x} x^{2 n-1},
$$


for $x \geq 0$. By noting that

$$
\begin{aligned}
\left(e^{x} f_{n}(x)\right)^{\prime} & =e^{x}\left(f_{n}(x)+f_{n-1}(x)\right) \\
& =e^{x} \cdot \frac{x^{n-1}}{(n-1) !}
\end{aligned}
$$

We compute

$$
\begin{aligned}
(L(x))^{\prime}= & 2 e^{2 x}((n-1) !)^{2} f_{n}(x) \cdot \frac{x^{n-1}}{(n-1) !}-e^{2 x}\left(x^{n}+n x^{n-1}\right)(n-1) ! \frac{x^{n-1}}{(n-1) !} \\
& -e^{2 x}\left(x^{n}+2 n x^{n-1}+n(n-1) x^{n-2}\right)(n-1) ! f_{n}(x) \\
& +2 e^{2 x} x^{2 n-1}+(2 n-1) e^{2 x} x^{2 n-2} \\
= & e^{2 x} x^{n-2}\left[(n-1) ! f_{n}(x)\left((2-2 n) x-x^{2}-n(n-1)\right)\right. \\
& \left.+x^{n+1}+(n-1) x^{n}\right] .
\end{aligned}
$$

Denote by

$$
l(x)=(n-1) ! f_{n}(x)\left((2-2 n) x-x^{2}-n(n-1)\right)+x^{n+1}+(n-1) x^{n}, \text { for } x \geq 0 .
$$

By a direct computation,

$$
\begin{aligned}
(l(x))^{(n)}= & -(n-1) ! \sum_{k=0}^{n}\left(\begin{array}{l}
n \\
k
\end{array}\right)\left(f_{n}(x)\right)^{(n-k)}\left(2(n-1) x+x^{2}+n(n-1)\right)^{(k)} \\
& +(n+1) ! x+(n-1) \cdot n ! \\
= & -(n-1) !\left[e^{-x}\left(2(n-1) x+x^{2}+n(n-1)\right)+n\left(1-e^{-x}\right)(2(n-1)\right. \\
& \left.+2 x)+n(n-1)\left(e^{-x}-1+x\right)\right]+(n+1) ! x+(n-1) \cdot n ! \\
= & (n-1) ! e^{-x}\left(2 x-x^{2}\right) .
\end{aligned}
$$

Thus

$$
(l(x))^{(n-1)}=(n-1) ! x^{2} e^{-x}>0, \quad \text { for } x>0 .
$$

Noting that

$$
l(0)=l^{\prime}(0)=\cdots=l^{(n-1)}(0)=0,
$$

we deduce that

$$
l(x)>0, \quad \text { for } x>0
$$


Hence by combining with (7.18) we know that

$$
\begin{aligned}
L(x) & =e^{2 x}\left[\left((n-1) ! f_{n}(x)\right)^{2}-(n-1) ! f_{n}(x)\left(x^{n}+n x^{n-1}\right)+x^{2 n-1}\right] \\
& >0, \quad \text { for } x>0 .
\end{aligned}
$$

So we have verified the inequality (D).

Summarizing above, we have shown that the complete Kähler metrics $g_{\alpha \bar{\beta}}=$ $\partial_{\alpha} \partial_{\bar{\beta}} u(t)$ obtained by solving (7.8) for each $\lambda>1$ with $c=(-1)^{n+1} n !(1-\lambda)$ have strictly positive curvature operator on the subspace of $(1,1)$-forms.

Finally we study the volume growth and the curvature decay of the Kähler metrics. The following asymptotic volume and curvature decay properties seem already known to Cao(see for example [3]). Since they haven't appear in literatures explicitly, we would like to include them as follows.

From the equation (7.8) one can see that $\phi$ tends to $+\infty$ as $t \rightarrow+\infty$ and

$$
t=\int \frac{\lambda d \phi}{\phi+O(1)}
$$

which implies that $\phi$ is asymptotic to $e^{\frac{t}{\lambda}}$ as $t \rightarrow+\infty$. And then from the equation $\phi^{\prime}$ is asymptotic to $\frac{1}{\lambda} e^{\frac{t}{\lambda}}$. Since the metric $g_{\alpha \bar{\beta}}=\partial_{\alpha} \partial_{\bar{\beta}} u(t)$ is rotationally symmetric, it is clear that the straight line through the origin are geodesics and the distance function $d$ from the origin $O$ is a function of $t$ only and is given by

$$
\begin{aligned}
d & =\int_{0}^{r} \sqrt{g_{1 \overline{1}}(r)} d r \\
& =\frac{1}{2} \int_{-\infty}^{t} \sqrt{\phi^{\prime}} d t
\end{aligned}
$$

by using (7.2). It then follows that the geodesic distance $d$ is asymptotic to $\sqrt{\lambda} e^{\frac{t}{2 \lambda}}$ as $t \rightarrow+\infty$. By (7.3), (7.4) and the asymptotic behaviors of $\phi$ and $\phi^{\prime}$ as $t \rightarrow+\infty$, we have the scalar curvature

$$
\begin{aligned}
R & =g^{\alpha \bar{\beta}} R_{\alpha \bar{\beta}} \\
& =\left(e^{t} \phi^{-1} \delta_{\alpha \beta}+\bar{z}_{\beta} z_{\alpha}\left(\left(\phi^{\prime}\right)^{-1}-\phi^{-1}\right)\right) \partial_{\alpha} \partial_{\bar{\beta}}\left(n t-(n-1) \log \phi-\log \phi^{\prime}\right) \\
& =O\left(\phi^{-1}\right) \\
& =O\left(\frac{1}{d^{2}}\right) .
\end{aligned}
$$


And by (7.4) and the asymptotic behavior of $\phi$ as $t \rightarrow+\infty$, we have the volume growth

$$
\begin{aligned}
\operatorname{Vol}(B(O, d)) & =\omega_{2 n-1} \int_{0}^{d} \operatorname{det}\left(g_{\alpha \bar{\beta}}\right) r^{2 n-1} d r \\
& =\frac{\omega_{2 n-1}}{2} \int_{-\infty}^{t} \phi^{\prime}(\phi)^{n-1} d t \\
& =\frac{\omega_{2 n-1}}{2 n} \phi^{n} \\
& =\frac{\omega_{2 n-1}}{2 n}\left(e^{\frac{t}{\lambda}}\right)^{n}+\text { lower order terms } \\
& =\frac{\omega_{2 n-1}}{2 n \lambda^{n}} d^{2 n}+\text { lower order terms, as } d \rightarrow+\infty,
\end{aligned}
$$

where $B(O, d)$ is the geodesic ball centered at the origin $O$ and of radius $d$ with respect to the Kähler metric $g_{\alpha \bar{\beta}}=\partial_{\alpha} \partial_{\bar{\beta}} u(t)$, and $\omega_{2 n-1}$ is the area of the unit sphere $S^{2 n-1}$. The standard volume comparison theorem thus gives

$$
\operatorname{Vol}(B(x, d)) \geq \frac{\omega_{2 n-1}}{2 n \lambda^{n}} d^{2 n}
$$

for any $x \in M, d>0$.

Therefore the complete Kähler metrics $g_{\alpha \bar{\beta}}=\partial_{\alpha} \partial_{\bar{\beta}} u(t)$ obtained by solving (7.8) for every $\lambda>1$ satisfy all the assumptions of Theorem 3.

\section{References}

[1] Berger, M., Sur les groupes d'holonomie des variétés à connexion affine et les variétés riemanniennes, Bull. Soc. Math. France, 83 (1955), 279-330.

[2] Cao, H. D., On Harnack's inequalities for the Kähler-Ricci flow, Invent. Math., 109 (1992), 247-263.

[3] Cao, H. D., Existence of gradient Kähler-Ricci solitons, Elliptic and Parabolic Methods in Geometry, Minnesota, (1994), 1-16.

[4] Cao, H. D., Limits of Solutions to the Kähler-Ricci flow, J. Differential Geom., 45 (1997), 257-272.

[5] Cao, H. D. and Chow, B., Compact Kähler manifolds with nonnegative curvature operator, Invent. Math., 83 (1986), 553-556. 
[6] Cheeger, J. and Gromoll, D., On the structure of complete manifolds of nonnegative curvature, Ann. of Math., 46 (1972), 413-433.

[7] Cheeger, J., Gromov, M. and Taylor, M., Finite propagation speed, kernel estimates for functions of the Laplace operator, and the geometry of complete Riemannian manifolds, J. Differential Geom., 17 (1982), 15-53.

[8] Chen, B. L., Tang, S. H. and Zhu, X. P., A uniformization theorem of complete noncompact Kähler surfaces with positive bisectional curvature, to appear in J. Differential Geometry.

[9] Chen, B. L. and Zhu, X. P., On complete noncompact Kähler manifolds with positive bisetional curvature, Math. Ann. 327(2003), no.1, 1-23.

[10] Demailly, J. P., $L^{2}$ vanishing theorems for positive line bundles and adjunction theory, Lecture Notes in Math., Vol. 1646, 1-97.

[11] Hamilton, R. S., Four-manifolds with positive curvature operator, J. Differential Geom. 24 (1986), 153-179.

[12] Hamilton, R. S., A compactness property for solution of the Ricci flow, Amer. J. Math., 117 (1995), 545-572.

[13] Hamilton, R. S., The formation of singularities in the Ricci flow, Surveys in Differential Geometry, 2 (1995), 7-136, International Press.

[14] Hörmander, L., $L^{2}$-estimates and existence theorem for the $\bar{\partial}$-operator, Acta Math., 113 (1965), 89-152.

[15] Klembeck, P., Complete Kähler metrics of positive holomorphic sectional curvature, Proc. Amer. Math. Soc., 64(1977), 313-317.

[16] Kobayashi, S. and Nomizu, K., Foundations of differential geometry, Vol. II, Interscience Publications, (1969).

[17] Li, P. and Schoen, R., $L^{p}$ and mean value properties of subharmonic functions on Riemannian manifolds, Acta Math., 153(1984), 279-301.

[18] Mok, N., Siu, Y. T., and Yau, S. T., The Poincaré-Lelong equation on complete Kähler manifolds, Compositio Math., 44 (1981), 183-218.

[19] Ni, L. and Tam, L. F., Liouville properties of plurisubharmonic functions, Preprint.

[20] Schoen, R. and Yau, S. T., Lectures on differential geometry, in Conference Proceedings and Lecture Notes in Geometry and Topology, Vol. 1, International Press Publications, (1994). 
[21] Shi, W. X., Deforming the metric on complete Riemannian manifold, J. Differential Geom., 30 (1989), 223-301.

[22] Shi, W. X., Ricci flow and the uniformization on complete noncompact Kähler manfolds, J. Differential Geom., 45 (1997), 94-220.

[23] To, W. K., Quasi-projective embeddings of noncompact complete Kähler manifolds of positive Ricci curvature and satisfying certain topological conditions, Duke Math. J., 63 (1991), 745-789.

[24] Wu, H., An elementary method in the study of nonnegative curvature, Acta Math., 142 (1979), 57-78.

[25] Yau, S. T., Some function-theoretic properties of complete Riemannian manifolds and their applications to geometry, Indiana Univ. Math. J. 25(1976), 659-670.

[26] Yau, S. T., A review of complex differential geometry, Proc. Symp. Pure Math., Vol. 52, Part II, Amer. Math. Soc., (1991).

Department of Mathematics, Zhongshan University,

Guangzhou 510275, P. R. China 